TEPP RAPPORT DE RECHERCHE

\title{
Grèves et productivité du travail : Application au cas français
}

\author{
JEREMY TANGUY
}

\section{www.tepp.eu}

TEPP - Travail, Emploi et Politiques Publiques - FR CNRS 3435 


\title{
Grèves et productivité du travail : application au cas français
}

\author{
Jeremy Tanguy*
}

\begin{abstract}
Résumé
L'objectif de cet article est d'apporter une première évaluation de l'effet de la fréquence des grèves sur la productivité du travail, dans le cadre des entreprises françaises, à partir des données appariées de l'enquête REPONSE 2004-2005 et des Enquêtes Annuelles d'Entreprises - EAE. Les travaux anglo-saxons présentent des arguments théoriques contradictoires sur cette question et conduisent à des résultats empiriques contrastés. En contrôlant le biais d'hétérogénéité inobservée, à travers l'utilisation d'une approche par fonction de contrôle, nous montrons que la fréquence des grèves a un effet nonlinéaire sur la productivité du travail des entreprises françaises, qui s'avère être positif et croissant jusqu'à un certain seuil, puis neutre au-delà. Nous mettons ensuite en évidence que l'effet des grèves sur la productivité du travail varie sensiblement en fonction de l'absentéisme des salariés dans l'entreprise. L'incidence de grèves peut affecter positivement et indirectement la productivité du travail, à condition de ne pas être associée à un problème d'absentéisme des salariés. A l'inverse, lorsqu'elles s'accompagnent de cette expression individuelle de conflit de la part salariés, les grèves ne présentent aucun effet sur la productivité du travail.
\end{abstract}

\begin{abstract}
The aim of this paper is to provide a first assessment of the effect of strike frequency on labor productivity, in the context of French firms, using merged data from the REPONSE 2004-2005 survey and the French Annual Business Survey - EAE. The Anglo-Saxon literature outlines contradictory theoretical arguments on this question and provides mixed empirical results. Controlling for the unobserved heterogeneity bias, using a control function approach, we show that strike frequency has a non-linear effect on French firms' labor productivity, which proves to be positive and growing up to a certain threshold, and non-significant beyond. Then, we highlight that the effect of strike frequency on labor productivity varies significantly depending on employees' absenteeism within the firm. Strike incidence may affect positively and indirectly labor productivity, provided not to be associated with a problematic absenteeism within the workforce. In contrast, when associated with this individual expression of conflict from employees, strikes have no effect on labor productivity.
\end{abstract}

Keywords : labor conflict; strikes ; labor productivity; endogeneity

JEL Classification : J52; C31; J24

*GAINS-TEPP, Université du Maine. E-mail : jeremy.tanguy@univ-lemans.fr. Adresse : UFR Droit, Economie et Gestion - Avenue Olivier Messiaen - 72 085 Le Mans Cedex 9. Tel : +33 (0)2 43833132.

Je tiens à remercier Patrick Musso, Mareva Sabatier, Claire Salmon, Yannick L'Horty, Patrice Laroche et JeanYves Lesueur pour leurs commentaires avisés. Je remercie également les participants aux conférences/séminaires JMA 2012, AFSE 2012 et GAINS 2013. Toute erreur restante est de la responsabilité de l'auteur. 


\section{Introduction}

La qualité des relations professionnelles entre salariés et employeur est reconnue, dans la littérature, comme un déterminant majeur de la performance économique des entreprises (e.g. Kleiner et al. 2002; Krueger et Mas 2004; Mas 2008). L'intensité des grèves a très souvent été utilisée pour évaluer la qualité des relations professionnelles (Flaherty 1987; Kleiner et al. 2002). L'intérêt dominant dans la littérature pour cette expression collective de conflit s'est justifié historiquement par sa forte visibilité et sa mesure consécutivement aisée par la statistique publique, à la différence d'expressions individuelles de conflit telles que l'absentéisme, l'indiscipline et les griefs des salariés (e.g. Ichniowski 1986; Katz et al. 1983 ; Kleiner et al. 1995 ; Norsworthy et Zabala 1985). Dans la littérature consacrée à l'effet des grèves sur la productivité des entreprises, le manque de données microéconomiques sur ces expressions individuelles de conflit a conduit les auteurs à interpréter leurs résultats empiriques en formulant des hypothèses contradictoires sur l'interaction entre ces différentes formes de conflit dans l'entreprise.

La nature de l'effet des grèves sur la productivité des entreprises a donné lieu à des résultats empiriques contrastés, dans la littérature anglo-saxonne. Plusieurs auteurs estiment un effet négatif des grèves sur la productivité du travail (e.g. Flaherty 1987), qu'ils interprètent en partie sur la base de leur complémentarité avec des formes individuelles de conflit, elles-mêmes néfastes pour la productivité (e.g. absentéisme). D'autres auteurs soulignent, à l'inverse, l'effet positif de l'activité de grève sur la productivité, par l'apport d'un mécanisme d'expression collective aux salariés, impliquant une amélioration de leur coopération sur le lieu de travail et un moindre recours à des formes individuelles de conflit (e.g. Drinkwater et Ingram 2005 ; Godard 1992 ; Knight 1989). Dans cette deuxième approche, les grèves et ces formes individuelles de conflit sont davantage conçues comme des expressions de conflit substituables dans l'entreprise. Bien que cette relation est reconnue comme centrale pour évaluer l'effet des grèves sur la productivité, elle n'a pas été considérée empiriquement dans cette littérature.

L'objectif de cet article est d'évaluer dans quelle mesure l'effet de la fréquence des grèves sur la productivité du travail varie en fonction du niveau d'absentéisme dans l'entreprise, dans le cadre français. Peu de travaux empiriques se sont intéressés à l'activité de grève en France et aucun, à notre connaissance, à son effet sur la productivité des entreprises. L'évaluation des conflits du travail ne s'est développée que récemment en France, contrairement à la plupart des pays anglo-saxons où, dans ce champ, les données microéconomiques sont historiquement plus répandues. La mise en oeuvre d'enquêtes d'entreprises sur le thème des relations professionnelles, en France, offre désormais des possibilités d'analyse comparables.

Nous utilisons, dans cet article, les données de l'enquête REPONSE 2004-2005 (Dares), que nous avons appariées avec les données des Enquêtes Annuelles d'Entreprises, EAE (Insee), afin de pouvoir relier la fréquence des grèves à une mesure objective de la productivité du travail. L'enquête REPONSE apporte, en outre, une information statistique sur plusieurs formes individuelles de conflit dans les entreprises interrogées et notamment l'absentéisme, que nous mobilisons dans cet article. Nous adoptons une approche par fonction de contrôle (e.g. Vella et Verbeek 1999), afin de tenir compte des biais potentiels d'endogénéité de la fréquence des grèves et de sélection endogène des entreprises en fonction de l'absentéisme.

Le reste de l'article s'articule comme suit : nous présentons les spécificités du contexte français et examinons la littérature existante au regard de notre question de recherche dans la 
section 2. Les données et variables sont présentées dans la section 3. L'estimation de l'effet de la fréquence des grèves sur la productivité du travail est discutée dans la section 4 et la section 5 analyse la variation de cet effet des grèves en fonction de l'absentéisme dans l'entreprise. La section 6 conclut.

\section{Contexte}

\subsection{Grèves et conflits du travail en France}

La grève se définit comme 'un arrêt de travail temporaire par un groupe de salariés afin d'exprimer un grief ou d'imposer une demande' (Hyman 1972, p. 17). La France fait partie des quelques pays où le droit de grève est garanti par le législateur et inscrit dans la constitution (Scheuer (2006), p. 145-146). Le déclenchement d'une grève n'est pas explicitement contraint en France, contrairement à d'autres pays comme la Grande-Bretagne, où l'action collective doit remporter la majorité des suffrages des salariés, lors d'un scrutin préalable, pour être légale. En France, seul le secteur public prévoit des aménagements à l'expression du droit de grève, à travers notamment l'obligation de déposer un préavis, au moins cinq jours avant le début de la grève, et l'obligation d'assurer un service minimum, chez certaines catégories de personnel. Dans le secteur privé, les salariés grévistes ne sont tenus qu'à une information préalable de l'employeur sur leurs revendications. En France, les employeurs ne peuvent ni licencier les salariés grévistes, ni embaucher des travailleurs temporaires pour les remplacer (Besancenot et Vranceanu 1999, p. 358).

Les statistiques nationales sur les grèves diffèrent sensiblement selon qu'elles proviennent des données administratives ou des données d'enquêtes ${ }^{1}$. Le recensement des grèves a, pendant longtemps, été assuré en France par les inspections du travail avec comme seul indicateur statistique le volume de 'journées individuelles non travaillées' (JINT). Celui-ci apparait avoir quasi-continuellement diminué depuis le milieu des années 1970, passant de 3500000 à 193000 , entre 1975 et 2004 (Carlier 2008). Des études statistiques ont montré que ces données administratives avaient largement sous-évalué l'ampleur réelle des grèves, et des conflits collectifs du travail de façon plus générale, en France (e.g. Brochard 2003; Carlier 2008). Ces données administratives se sont avérées défaillantes dans le recensement des conflits du travail de courte durée et des conflits généralisés ${ }^{2}$. À cet égard, la France a observé des regains exceptionnels de la grève programmée, des journées nationales d'action et des mouvements interprofessionnels en 1995 et en 2003 (Béroud et al. 2008, p. 224). Il apparaît, cependant, que ces conflits 'généralisés' sont principalement concentrés dans les fonctions publiques et les grandes entreprises publiques (Pernot 2005; Sirot 2002), et que l'essentiel des grèves est constitué depuis plusieurs années par des conflits localisés ${ }^{3}$ (Groux et Pernot

1. Enquête ACEMO sur le dialogue social en entreprise, anciennement intitulée 'enquête sur la négociation et la représentation des salariés', et REPONSE, réalisées par la Dares, Ministère du Travail, de l'Emploi et de la Santé.

2. Le conflit généralisé se définit comme 'toute cessation collective de travail résultant d'un mot d'ordre commun à plusieurs établissements appartenant à des entreprises différentes ou à plusieurs entreprises journées nationales d'action, grèves sectorielles ou pluri-sectorielles dans une région -' (Brochard 2003, p. 5).

3. Est considéré comme conflit localisé 'toute cessation collective du travail résultant d'un mot d'ordre interne à un établissement quelconque, ou plusieurs établissements d'une même entreprise' (Brochard 2003, p. 
2008, p. 81).

En France, les données d'enquêtes révèlent une augmentation sensible de la part des établissements concernés par au moins un conflit collectif entre 1996-1998 et 2002-2004, de $21 \%$ à $30 \%$ (Amossé 2006, p. 6). La part des établissements français concernés par des arrêts collectifs du travail a, quant à elle, augmenté de 2.4 points de pourcentage entre ces deux périodes (Béroud et al. 2008), pour atteindre $15 \%$ en 2004. Seule la part des établissements concernés par des grèves dites 'longues' - de deux jours et plus - a décliné ces dernières années. Les études récentes, menées dans le contexte français, montrent, en outre, que 'la grève s'organise rarement de façon exclusive et cohabite toujours avec d'autres formes de mobilisation' (Groux et Pernot 2008, p. 84). Les statistiques descriptives révèlent notamment que les expressions collectives et individuelles sont, de plus en plus, associées dans les établissements français. A partir des enquêtes REPONSE, Carlier et Tenret (2007) montrent notamment que la part des établissements français enregistrant à la fois des sanctions disciplinaires et des formes collectives de conflit ${ }^{4}$ est passé de $15.7 \%$ sur la période 1996-1998 à $23.7 \%$ sur la période $2002-2004$.

\section{$2.2 \quad$ Revue de la littérature}

La grève est la forme de conflit du travail qui a suscité le plus de travaux dans la littérature économique. Relativement à la littérature très étendue sur les déterminants de la grève, l'étude de ses effets sur la productivité a donné lieu à peu de travaux empiriques. Les auteurs s'accordent généralement sur l'existence d'effets directs négatifs de la grève (e.g. Flaherty 1987 ; McHugh 1991). La grève a un effet direct négatif, essentiellement de court terme, sur la productivité des firmes, car source de perturbations dans le processus de production. Ces perturbations peuvent prendre la forme, durant la grève, de 'tensions techniques et sociales' ou de 'goulots d'étranglement' (Flaherty 1987, p. 588), notamment lorsque seule une fraction de la main d'œuvre est concernée par la grève. La productivité de la firme peut également être altérée si les salariés obtiennent, à travers la grève, une restriction des initiatives de l'employeur ou la mise en oeuvre de règles de travail restrictives. En outre, en cherchant à accumuler des stocks, avant ou après la grève, la firme peut faire face à des contraintes de capacité, néfastes pour sa productivité. Une telle accumulation des stocks peut néanmoins s'avérer bénéfique pour la productivité si la firme parvient à atteindre un taux plus efficient d'utilisation de ses capacités de production. Ce mécanisme de réaction des firmes pourrait ainsi expliquer l'effet faiblement négatif voire neutre des grèves sur la productivité, qu'estiment certains auteurs (McHugh 1991, p. 722).

Au-delà de ces effets directs, la littérature anglo-saxonne présente des visions théoriques divergentes quant à la nature des effets indirects de la grève sur la productivité des entreprises. La vision dominante considère la fréquence des grèves comme un indicateur pertinent de la qualité des relations professionnelles. Dans cette vision, les grèves seraient simplement des 'manifestations extérieures d'un malaise, qui se manifesterait dans l'établissement sous la forme d'un faible moral des salariés, de taux croissants d'absentéisme, de ralentissements et

5)

4. Les formes collectives de conflit incluent la grève traditionnelle (inférieure, égale ou supérieure à deux jours), le débrayage, la pétition, la manifestation, la grève perlée, la grève du zèle ou le refus d'heures supplémentaires. 
du refus des salariés de coopérer volontairement avec l'employeur sur plusieurs aspects de la production' (Fairris 1998, p. 321). Ainsi, la grève s'apparente à la partie émergée de l'iceberg', en s'accompagnant d'expressions de conflit inorganisées et moins visibles, comme l'absentéisme (voir Sapsford et Turnbull 1994). Dans cette perspective, l'effet estimé de la fréquence des grèves sur la productivité du travail intègrerait l'effet négatif de telles expressions individuelles de conflit. Naples (1988) montre, dans ce sens, que le taux de grève et le taux de démission n'influent pas sur la productivité lorsque considérés conjointement alors que chacun présente séparément un effet négatif, et cela en raison de leur forte corrélation.

Cette vision dominante est néanmoins discutée à la lumière de résultats empiriques contradictoires. Knight (1989) met notamment en évidence un effet positif et croissant de la fréquence des grèves sur la productivité du travail, et ce jusqu'à un certain seuil, dans le cadre des industries manufacturières britanniques. Knight associe ce résultat empirique au 'rôle thérapeutique' de la grève, à travers l'apport d'un 'mécanisme permettant de résoudre des conflits qui autrement resteraient non-résolus' (p. 370). Cette vision alternative, empruntée à l'école d'Harvard, considère la grève comme une forme d'expression collective - ou 'collective voice' -, c'est-à-dire comme un moyen pour les salariés d'exprimer collectivement leur mécontentement et leur méfiance (Godard 1992, p. 340-341). Pour l'école d'Harvard, cette expression collective constitue l'un des 'visages' du syndicalisme et représente un moyen efficace de transmettre à l'employeur l'information détenue par les salariés, en vue d'améliorer la productivité (voir Freeman et Medoff 1979, 1984). Une meilleure communication dans l'entreprise permettrait, en outre, d'améliorer le 'moral' des salariés et leur coopération (Mefford 1986, p. 106). Farrell et Rusbult (1992) montrent notamment qu'à la différence de l'absentéisme et de l'indiscipline, l'expression collective est associée à des niveaux plus élevés de satisfaction et d'investissement dans le travail (voir Hebdon et Stern 1998, p. 206). Les preuves empiriques révèlent, en outre, que les grèves tendent à se substituer aux expressions individuelles de conflit des salariés (e.g. Hebdon et Stern 1998 ; Sapsford et Turnbull 1994; Tanguy 2013).

Ces visions opposées suggèrent une variation de l'effet des grèves sur la productivité du travail, selon la nature de leur relation - complémentarité ou substitution - avec l'expression individuelle de conflit des salariés. Cette variation de l'effet des grèves n'a pourtant pas été évaluée empiriquement, à notre connaissance, dans la littérature. Nous proposons, dans cet article, d'estimer l'effet de la fréquence des grèves sur la productivité du travail et d'évaluer dans quelle mesure cet effet varie en fonction de l'absentéisme des salariés dans l'entreprise.

\section{Données et variables}

\subsection{Données}

Dans cet article, nous utilisons deux sources de données individuelles : l'enquête RElations PrOfessionnelles et NégociationS d'Entreprises (REPONSE) et les Enquêtes Annuelles d'Entreprises (EAE). L'enquête REPONSE est une enquête réalisée conjointement par la Dares et l'Insee. Cette enquête fournit une information détaillée sur l'organisation et la qualité des relations professionnelles - i.e. représentation collective des salariés, négociation collective et conflits du travail. Réalisée à intervalles de temps réguliers (1992-1993, 1998-1999, 20042005, 2010-2011), cette enquête se compose de trois volets : 'représentants de la direction', 
'représentants du personnel' et 'salariés'. Nous utilisons, dans cet article, les données en coupe transversale issues du volet 'représentants de la direction' de l'édition 2004-2005, portant sur un échantillon de 2930 établissements, représentatif de l'ensemble des établissements de 20 salariés ou plus du secteur marchand non-agricole.

Les EAE sont des enquêtes réalisées par l'INSEE jusqu'en 2007, au niveau des entreprises françaises. Les EAE fournissent les informations comptables des entreprises issues de l'ensemble des secteurs marchands de l'économie française ${ }^{5}$. Les informations fournies par les EAE nous permettent, dans cet article, de déterminer objectivement la productivité du travail de chaque entreprise, ce que ne permet pas l'enquête REPONSE. Les données issues de REPONSE et des EAE ont ainsi été appariées grâce au numéro SIREN des entreprises référencées dans les deux sources, donnant lieu à un échantillon cylindré de 2149 observations.

L'étude conjointe de données d'établissement et d'entreprise peut poser problème pour les entreprises multi-établissements, compte tenu de l'écart entre l'unité d'analyse des variables explicatives et celle de la variable expliquée (Black et Lynch 2001, p. 263). Le principal risque, pour ces entreprises, est que les variables d'établissement ne soient pas de bonnes indicatrices des variables d'entreprise, impliquant une sous-estimation de leur coefficient dans les régressions (Breda 2008, p. 9). Toutefois, quelques études révèlent que, dans le cadre de l'enquête REPONSE, les variables d'établissement sont des indicatrices valides de la situation au niveau de l'entreprise (e.g. Breda 2008; Coutrot 1996) et inversement (e.g. Ananian et Aubert 2006). Aussi, nous pouvons montrer, à l'instar des résultats d'Ananian et Aubert (2006) en $1998^{6}$, que les établissements de l'échantillon REPONSE 2004-2005 représentent, en moyenne, une part importante dans l'emploi total de leur entreprise : 48,7\% des établissements de l'échantillon sont des entreprises mono-établissements et le poids moyen des établissements au sein de leur entreprise est de $64,6 \%$.

\subsection{Variables d'intérêt}

Dans cet article, nous mesurons la productivité du travail grâce à la valeur ajoutée par salarié. Ce type de mesure basée sur la valeur ajoutée a été retenue dans quelques études consacrées à l'effet des grèves (e.g. Bemmels 1987; Knight 1989; McHugh 1991). Par rapport à son alternative basée sur la production, cette mesure a l'avantage d'ignorer l'impact des variations d'intrants intermédiaires entre les entreprises, qui rendent les mesures de productivité basées sur la production assez 'bruyantes' dans certains secteurs (Syverson 2004, p. 539). En utilisant une mesure basée sur la valeur ajoutée, la valeur des intrants intermédiaires est ainsi soustraite, permettant de contrôler les différences de méthodes de production entre les secteurs (Jensen et al. 2001, p. 328). Puisque les intrants intermédiaires sont soustraits de la production nette pour obtenir la valeur ajoutée, ils n'intègrent pas le vecteur de variables explicatives dans l'équation de productivité. Ainsi, la valeur ajoutée est uniquement reliée aux intrants primaires (capital et travail), dans le cadre d'une fonction de production. Ce choix est, en outre, pertinent compte tenu du caractère potentiellement endogène des intrants

5. Les EAE se répartissent en six grands secteurs : industrie, services, commerce, transport, construction, Industries Agricoles et Alimentaires (IAA).

6. Ananian et Aubert (2006) mettent en évidence que 41\% des établissements de l'échantillon sont des entreprises mono-établissements et que le poids moyen des établissements au sein de leur entreprise est de $64 \%$ en termes de journées travaillées. 
intermédiaires (Amiti et Wei 2009, p. 208).

La fréquence des grèves est évaluée grâce à l'enquête REPONSE; l'édition 2004-2005 de cette enquête recense, pour la première fois, le nombre de grèves enregistrées au cours de la période considérée alors que les éditions précédentes - 1992-1993, 1998-1999 - se limitaient à leur seule présence. Deux formes de grèves sont dissociées en fonction de leur durée dans REPONSE : la grève 'courte' inférieure à deux jours et la grève 'longue' égale ou supérieure à deux jours. La fréquence de chaque forme de grève est exprimée par une variable ordinale, dont les modalités représentent le nombre de grèves enregistrées sur la période 2002-2004 sous la forme des intervalles suivants : 0,1 ou 2, de 3 à 5, plus de 5. Compte tenu du faible nombre d'observations enregistrées pour certaines fréquences, lorsque grèves 'courtes' et grèves 'longues' sont dissociées, et de l'association fréquente de ces deux formes de grèves dans les établissements français (voir Table 1), nous retenons une mesure agrégée de la fréquence des grèves, indépendamment de leur durée. La distribution de cette variable et les caractéristiques moyennes des entreprises pour chaque fréquence de grève sont présentées dans la Table 2.

TABLE 1 - Fréquences des grèves

\begin{tabular}{|c|c|c|c|c|c|c|}
\hline & & \multicolumn{5}{|c|}{ Fréquence des grèves $\geqslant 2$ jours } \\
\hline & & 0 & $1-2$ & $3-5$ & $>5$ & Total \\
\hline \multirow{4}{*}{ Fréquence des grèves $<2$ jours } & 0 & 1618 & 67 & 1 & 4 & 1690 \\
\hline & 1-2 & 227 & 29 & 4 & 0 & 260 \\
\hline & $3-5$ & 57 & 17 & 5 & 1 & 80 \\
\hline & $>5$ & 60 & 28 & 6 & 25 & 119 \\
\hline Total & & 1962 & 141 & 16 & 30 & 2149 \\
\hline
\end{tabular}

\subsection{Variables de contrôle}

La fonction de production, considérée dans cet article, tient compte de l'intensité capitalistique des entreprises à travers le ratio entre le stock de capital et le nombre total de salariés dans l'entreprise, sous forme logarithmique. La valeur comptable des immobilisations corporelles est utilisée ici comme mesure approximative du stock réel de capital ${ }^{7}$. Le logarithme du nombre total de salariés est également introduit afin de tenir compte des variations de productivité en fonction de la taille de l'entreprise (e.g. Haltiwanger et al. 1999). Nous contrôlons les effets fixes sectoriels en introduisant un ensemble de variables binaires, chacune indiquant l'appartenance de l'entreprise à l'un des 16 postes de la Nomenclature Economique de Synthèse (NES). L'âge de l'entreprise est également contrôlé (voir, e.g., Jensen et al. 2001), à travers un ensemble de variables binaires, chacune correspondant à une des six classes d'âge définies dans la Table 2. Enfin, nous contrôlons aussi les entreprises cotées en bourse et les entreprises multi-établissements à travers deux variables binaires. La littérature existante

7. De nombreux travaux prônent pour l'utilisation de la méthode de l'inventaire permanent pour évaluer le stock de capital. Dans notre cas, l'utilisation d'une telle mesure génère cependant un nombre important de valeurs manquantes et réduit sensiblement la taille de notre échantillon. 
TABLE 2 - Statistiques descriptives

\begin{tabular}{|c|c|c|c|c|c|}
\hline \multirow[b]{3}{*}{ Variables } & \multicolumn{5}{|c|}{ Fréquence des grèves } \\
\hline & 0 & $1-2$ & $3-5$ & $>5$ & $\geqslant 1$ \\
\hline & Moy. & Moy. E.-T. & Moy. E.-T. & Moy. E.-T. & Moy. E.-T. \\
\hline $\ln y$ & $3.852(0.604)$ & $4.048 \quad(0.616)$ & $4.144 \quad(0.703)$ & $4.352 \quad(0.769)$ & $4.141 \quad(0.684)$ \\
\hline $\ln K / L$ & $3.358(1.456)$ & $4.069 \quad(1.514)$ & $3.867 \quad(1.850)$ & $5.106 \quad(1.763)$ & $4.280 \quad(1.713)$ \\
\hline $\ln L$ & $5.909(1.979)$ & $6.834(1.681)$ & $6.881 \quad(1.367)$ & $8.624 \quad(1.955)$ & $7.279 \quad(1.855)$ \\
\hline$C S P$ & & & & & \\
\hline$\%$ cadres & $0.158 \quad(0.205)$ & $0.143(0.176)$ & $0.168 \quad(0.206)$ & $0.209 \quad(0.211)$ & $0.164 \quad(0.193)$ \\
\hline$\%$ TAM & $0.194 \quad(0.158)$ & $0.229 \quad(0.162)$ & $0.208 \quad(0.145)$ & $0.330 \quad(0.198)$ & $0.249 \quad(0.174)$ \\
\hline \% employés & $0.226 \quad(0.288)$ & $0.167 \quad(0.243)$ & $0.112(0.164)$ & $0.085 \quad(0.095)$ & $0.136 \quad(0.204)$ \\
\hline$\%$ ouvriers & $0.422 \quad(0.332)$ & $0.461 \quad(0.299)$ & $0.512(0.296)$ & $0.377 \quad(0.291)$ & $0.451 \quad(0.300)$ \\
\hline$\%$ femmes & $0.347 \quad(0.238)$ & $0.306 \quad(0.205)$ & $0.238 \quad(0.177)$ & $0.217 \quad(0.154)$ & $0.270 \quad(0.192)$ \\
\hline$\% \mathrm{CDD}$ & $0.052 \quad(0.117)$ & $0.033(0.071)$ & $0.048 \quad(0.114)$ & $0.028 \quad(0.093)$ & $0.035 \quad(0.087)$ \\
\hline Présence syndicale & 0.685 & 0.976 & 0.991 & 1.000 & 0.985 \\
\hline Absentéisme & 0.511 & 0.599 & 0.593 & 0.566 & 0.589 \\
\hline Multi-établissements & 0.596 & 0.667 & 0.643 & 0.806 & 0.719 \\
\hline Bourse & 0.374 & 0.588 & 0.556 & 0.465 & 0.552 \\
\hline \multicolumn{6}{|l|}{ Age entreprise } \\
\hline$<10$ ans & 0.153 & 0.194 & 0.176 & 0.062 & 0.158 \\
\hline $10-14$ ans & 0.135 & 0.150 & 0.204 & 0.124 & 0.154 \\
\hline $15-19$ ans & 0.169 & 0.139 & 0.148 & 0.085 & 0.128 \\
\hline $20-29$ ans & 0.209 & 0.122 & 0.148 & 0.140 & 0.132 \\
\hline $30-44$ ans & 0.149 & 0.146 & 0.111 & 0.101 & 0.128 \\
\hline 45 ans et plus & 0.185 & 0.248 & 0.213 & 0.488 & 0.299 \\
\hline \multicolumn{6}{|l|}{ Secteur (NES 16) } \\
\hline Agriculture & 0.000 & 0.000 & 0.000 & 0.000 & 0.000 \\
\hline IAA & 0.054 & 0.054 & 0.019 & 0.031 & 0.041 \\
\hline Industrie - biens de conso. & 0.071 & 0.078 & 0.102 & 0.039 & 0.073 \\
\hline Industrie automobile & 0.016 & 0.027 & 0.028 & 0.031 & 0.028 \\
\hline Industrie - biens d'équipement & 0.082 & 0.129 & 0.130 & 0.186 & 0.143 \\
\hline Industrie - biens intermédiaires & 0.153 & 0.296 & 0.287 & 0.202 & 0.271 \\
\hline Energie & 0.015 & 0.041 & 0.037 & 0.248 & 0.090 \\
\hline Construction & 0.107 & 0.027 & 0.019 & 0.000 & 0.019 \\
\hline Commerce & 0.174 & 0.116 & 0.065 & 0.008 & 0.079 \\
\hline Transports & 0.054 & 0.068 & 0.176 & 0.155 & 0.111 \\
\hline Activités financières & 0.004 & 0.000 & 0.000 & 0.000 & 0.000 \\
\hline Activités immobilières & 0.016 & 0.020 & 0.000 & 0.000 & 0.011 \\
\hline Services aux entreprises & 0.212 & 0.119 & 0.102 & 0.070 & 0.104 \\
\hline Services aux particuliers & 0.042 & 0.024 & 0.037 & 0.031 & 0.028 \\
\hline Education, santé, action sociale & 0.000 & 0.000 & 0.000 & 0.000 & 0.000 \\
\hline Administration & 0.000 & 0.000 & 0.000 & 0.000 & 0.000 \\
\hline \multicolumn{6}{|l|}{ Instruments } \\
\hline Taux de syndicalisation $>5 \%$ & 0.370 & 0.690 & 0.741 & 0.829 & 0.734 \\
\hline Faible activité syndicale (bassin d'emploi) & 0.539 & 0.442 & 0.250 & 0.310 & 0.371 \\
\hline CFDT & 0.488 & 0.816 & 0.824 & 0.946 & 0.849 \\
\hline CGT & 0.485 & 0.840 & 0.926 & 0.969 & 0.889 \\
\hline Observations & 1618 & 294 & 108 & 129 & 531 \\
\hline
\end{tabular}

Notes : Ecarts-types entre parenthèses dans le cas des variables continues 
souligne, à cet égard, que les entreprises cotées en bourse sont plus productives que celles qui ne le sont pas (DeVaro 2008) alors que les entreprises mono-établissements présentent des niveaux de productivité du travail inférieurs aux entreprises multi-établissements (Zwick 2004).

Dans le cadre de cette fonction de production augmentée, nous introduisons certaines caractéristiques de la main d'oeuvre susceptibles d'expliquer significativement des variations de productivité du travail entre les entreprises (voir, e.g., Haltiwanger et al. 1999). Nous tenons compte notamment de la structure des emplois, en introduisant la part de chaque catégorie socio-professionnelle (CSP) - cadres, techniciens et agents de maitrise (TAM), employés, ouvriers - au sein de la main d'oeuvre. La littérature empirique existante montre, à cet égard, que la productivité du travail augmente significativement avec le pourcentage de travailleurs qualifiés dans la main d'oeuvre (e.g. Black et Lynch 2001 ; Matteucci et al. 2005). Un certain nombre de travaux prennent en considération la part des salariés à temps partiel dans la main d'oeuvre. Cette information n'est malheureusement pas disponible dans REPONSE 2004-2005. Nous disposons néanmoins du pourcentage de femmes dans la main d'oeuvre; la prise en compte de cette variable est susceptible de capter, pour partie, l'effet de l'emploi à temps partiel sur la productivité du travail, compte tenu de la sur-représentation de cette forme de contrat de travail parmi les femmes (e.g. Buffeteau et Essafi 2006) ${ }^{8}$. En outre, la préférence des femmes pour des activités permettant une plus grande flexibilité entre leur emploi et leur vie de famille, et par conséquent leur plus faible interdépendance avec les autres salariés, peuvent également expliquer une plus faible productivité du travail dans les entreprises enregistrant une forte proportion de femmes (Zwick 2004, p. 724). Au regard de la structure des contrats, nous prenons en compte la part des salariés en contrat à durée déterminée (CDD), potentiellement liée à la productivité du travail de par sa corrélation avec l'effort de travail. En effet, la littérature reconnait généralement que les salariés disposant d'un contrat temporaire tendent à fournir un effort plus important que les salariés permanents, dans l'optique d'une ré-embauche future, bien que ces contrats précaires soient sources de démoralisation et de moindre engagement des salariés dans l'entreprise (Green 2004, p. 720).

Enfin, la fréquence des grèves étant particulièrement liée à la présence syndicale en France (voir Amossé et Jacod 2008), nous introduisons une variable binaire indiquant qu'au moins un délégué syndical est présent dans l'entreprise afin d'isoler l'effet de la présence syndicale dans l'effet estimé de la fréquence des grèves. Nous évaluons ainsi l'effet autonome des syndicats sur la productivité du travail, sachant que la grève est considérée comme un des canaux de l'effet syndical sur la productivité (voir, e.g., Bemmels 1987).

\subsection{Indicateur d'absentéisme}

Dans l'enquête REPONSE 2004-2005, l'information relative à l'absentéisme est limitée puisqu'elle résulte de la perception des représentants de la direction sur l'aspect problématique ou non de l'absentéisme pour les catégories professionnelles représentées dans l'entreprise. ${ }^{9}$

8. En 2002 , près de $30 \%$ des femmes occupaient un emploi à temps partiel, contre seulement $6 \%$ des hommes (Buffeteau et Essafi 2006).

9. La question posée aux représentants de la direction est la suivante : "En 2004, l'absentéisme a-t-il représenté pour vous un problème chez (a) les cadres, (b) les commerciaux, (c) les techniciens et agents de maîtrise (TAM), (d) les employés, (e) les ouvriers." 
Nous utilisons cette information pour construire une variable binaire, égale à 1 si l'employeur fait face à un problème d'absentéisme pour au moins une catégorie professionnelle dans l'entreprise, égale à 0 sinon. La distribution de cette variable, dans l'échantillon total et en fonction de la fréquence de grèves dans l'entreprise, est présentée respectivement dans les Tables 7 et 2. Dans notre échantillon, 53.1\% des entreprises reportent un problème d'absentéisme pour au moins une des catégories professionnelles représentées dans leur main d'oeuvre. Cette proportion varie légèrement entre les entreprises grévistes et non-grévistes sur la période 2002-2004, soit respectivement 51.1\% et 58.9\%. Entre outre, la part des entreprises grévistes concernées par un problème d'absentéisme diminue avec la fréquence des grèves sur la période 2002-2004.

\section{Effet des grèves sur la productivité du travail}

L'équation de productivité du travail que nous souhaitons estimer est de la forme suivante :

$$
\ln y_{i}=\mathbf{X}_{i}^{\prime} \beta+\sum_{j=1}^{3} \delta_{j} S_{i j}+u_{i}
$$

où $\ln y$ est la variable dépendante observée de productivité du travail, $\mathbf{X}^{\prime} \beta$ est une fonction linéaire des variables de contrôle observées $\mathbf{X}$, décrites dans la section $3.3, S_{j}$ sont des variables binaires, chacune étant égale à 1 si la fréquence de grèves $j$ a été enregistrée dans l'entreprise, égale à 0 sinon. $\delta_{j}$ représente l'effet de chaque fréquence de grèves $j$ sur le logarithme de la productivité du travail et $u$ correspond au terme d'erreur. Lorsque $E\left(u_{i} \mid \mathbf{X}_{i}, S_{i j}\right)=0$, les paramètres $\delta_{j}$ peuvent être estimés sans biais par la méthode des Moindres Carrés Ordinaires (MCO après). Plusieurs raisons peuvent cependant justifier l'existence d'une corrélation nonnulle entre les fréquences de grèves $S_{j}$ et le terme d'erreur $u$ dans l'équation de productivité du travail.

On peut, tout d'abord, supposer que le terme d'erreur $u_{i}$ dans l'équation (1) se compose de deux éléments : $u_{i}=\alpha_{i}+\epsilon_{i}$, où $E\left(\alpha_{i} \mid \mathbf{X}_{i}, S_{i j}\right) \neq 0$ et $\epsilon_{i}$ intègre des facteurs inobservés non-corrélés avec l'ensemble des variables introduites dans l'équation de productivité du travail. On peut, en effet, escompter que les entreprises dotées d'une plus grande capacité productive ont à la fois un niveau plus élevé de productivité du travail et une fréquence plus élevée de grèves. La littérature présente des exemples de facteurs inobservés expliquant à la fois l'incidence de grèves et le niveau de productivité dans l'entreprise. Notamment, des initiatives réussies de l'employeur pour accroître la productivité peuvent entraîner, selon Flaherty (1987), l'émergence de grèves défensives dont l'objectif est de freiner l'accélération de la productivité, de réclamer des règles ou pratiques de travail abandonnées. Selon Katz et al. (1983), la rotation de l'encadrement, les caractéristiques politiques de l'employeur et des syndicats, les différences de combinaison technologique et la répartition des compétences sont également des sources de variation à la fois de la productivité et des conflits du travail. La satisfaction, la motivation ou le 'moral' des salariés sont aussi des facteurs inobservés, susceptibles d'influencer négativement la productivité du travail et positivement la fréquence des grèves (e.g. Norsworthy et Zabala 1985).

Ensuite, l'effet de chaque fréquence de grèves $j$ sur la productivité du travail peut ne pas être homogène entre les entreprises mais, à l'inverse, varier en fonction de caractéristiques 
inobservables. On peut alors supposer que l'effet individuel de la fréquence de grèves $j$ sur la productivité du travail pour l'entreprise $i$ se décompose ainsi : $\delta_{j}+\delta_{i j}$, où $\delta_{i j}$ désigne une composante idiosyncratique de l'effet de la fréquence de grèves $j$ associée à l'entreprise $i$. Dans ce cas précis, nous avons une distribution des paramètres $\delta_{i j}$ et il y a un biais lorsque $E\left(\delta_{i j} \mid \mathbf{X}_{i}, S_{i j}\right) \neq 0$. L'utilisation d'une variable agrégée de fréquence des grèves - i.e. grèves courtes et grèves longues ${ }^{10}$ - peut être en partie source d'un tel biais d'hétérogénéité. En effet, l'effet des fréquences de grèves est susceptible de varier en fonction de la durée des grèves (e.g. Knight 1989). De plus, les fréquences de grèves sont exprimées, dans l'enquête REPONSE, sous forme d'intervalles : 1-2, 3-5, > 5 grèves. L'hétérogénéité de l'effet d'une fréquence donnée de grèves est ainsi d'autant plus probable que l'intervalle défini est large.

Nous adoptons une approche par fonction de contrôle (e.g. Vella et Verbeek 1999)) afin de contrôler cette sélection potentielle sur les caractéristiques inobservables. Cette approche permet de purger le terme d'erreur $u_{i}$ des facteurs inobservés $\alpha_{i}$ communs à la fréquence des grèves et à la productivité du travail, de sorte que ce terme d'erreur n'intègre que les facteurs inobservés $\epsilon_{i}$ propres à la productivité du travail. Cette approche permet ainsi d'éliminer la corrélation non-nulle entre les variables de fréquence des grèves et le terme d'erreur $u_{i}$ dans l'équation de productivité du travail. Pour cela, l'approche par fonction de contrôle comprend deux étapes : la première étape permet de capter les facteurs inobservés de la fréquence des grèves en utilisant l'information contenue dans le terme d'erreur d'une équation de fréquence des grèves; cette information est ensuite introduite sous la forme de régresseurs additionnels, appelés 'résidus généralisés', dans l'équation de productivité du travail. Ces résidus généralisés permettent d'incarner les facteurs inobservés de la fréquence des grèves et ainsi de les extraire du terme d'erreur $u_{i}$, dans l'équation de productivité du travail. En première étape, nous estimons l'équation de fréquence des grèves suivante, grâce un modèle probit ordonné :

$$
S_{i}^{*}=\mathbf{W}_{i}^{\prime} \theta+\nu_{i}, \quad \nu_{i} \sim N(0,1)
$$

où $S^{*}$ est la variable latente non-observée de fréquence des grèves, $\mathbf{W}^{\prime} \theta$ est une fonction linéaire de variables exogènes observées $\mathbf{W}$, incluant les variables de contrôle $\mathbf{X}$ introduites dans l'équation (1) ainsi que d'autres variables $\mathbf{Z}$ non-introduites dans l'équation (1), appelées 'instruments', nécessaires pour que le modèle soit identifié autrement que par seule forme fonctionnelle ${ }^{11}$. Ces variables $\mathbf{Z}$ déterminent significativement $S^{*}$ mais n'ont pas d'effet direct non-nul sur $\ln y: E\left(u_{i} \mid \mathbf{Z}_{i}\right)=E\left(u_{i}\right)=0$. Le premier instrument retenu indique que le taux de syndicalisation de la main d'oeuvre est supérieur à 5\%. Les preuves empiriques montrent, en effet, que ce taux d'adhésion syndicale tend à accroître la probabilité de grève car les salariés syndiqués sont plus enclins à suivre la grève (Gramm 1986, p. 368). En outre, un fort taux de syndicalisation tend à renforcer la position de négociation des représentants syndicaux (Tracy 1986, p. 427). Le deuxième instrument indique que l'activité syndicale dans le bassin d'emploi est peu ou très peu intense. Dans la littérature, l'environnement de l'entreprise est reconnu jouer un rôle important dans l'émergence de grèves. Nicolitsas (2000) souligne notamment

10. Une mesure commune à ces deux formes de grèves est privilégiée, dans notre cas, en raison du nombre réduit d'observations pour certaines fréquences de grèves et de l'association fréquente de ces formes de grèves dans les établissements (voir Table 1).

11. D'après Heckman et Robb (1985), l'identification des paramètres est permise grâce à la non-linéarité du modèle probit ordonné et ne nécessite pas le recours à des instruments - ou restrictions d'exclusion. 
qu'au Royaume-Uni la fréquence des grèves augmenterait avec la couverture syndicale au niveau du secteur.

La variable latente $S^{*}$ est supposée être liée à la variable ordinale observée $S$ par la relation suivante :

$$
S_{i}=j \quad \text { si } \quad \mu_{j-1}<S_{i}^{*} \leqslant \mu_{j}, \quad j \in\{0,3\}, \quad \mu_{0}=-\infty, \quad \mu_{4}=+\infty
$$

où les paramètres de seuil $\mu_{j}$ sont inconnus et estimés en même temps que $\theta$, par maximum de vraisemblance. En supposant $\nu$ normalement distribué, la distribution conditionnelle de $S$ dans le cadre du modèle probit ordonné est donnée par :

$$
\operatorname{Pr}\left(S_{i}=j \mid \mathbf{W}_{i}\right)=\Phi\left(\mu_{j}-\mathbf{W}_{i} \theta\right)-\Phi\left(\mu_{j-1}-\mathbf{W}_{i} \theta\right)
$$

où $\Phi$ est la fonction de distribution cumulative de la loi normale standard. Les estimations de cette première étape, reportées dans la Table 3 , révèlent que les deux instruments retenus $\mathbf{Z}$ présentent bien des effets statistiquement significatifs sur la fréquence des grèves. Le calcul des effets marginaux, également reportés dans la Table 3, permet de vérifier que ces instruments influencent significativement la probabilité de chaque fréquence de grèves.

L'équation de productivité du travail augmentée, que nous estimons en deuxième étape, est de la forme suivante :

$$
\ln y=\mathbf{X}_{i}^{\prime} \beta+\sum_{j=1}^{3} \delta_{j} S_{i j}+\sigma_{\alpha} \sum_{j=0}^{3} S_{i j} \cdot \lambda_{i j}+u_{i}
$$

où $\lambda_{i j}(\cdot)$ sont les résidus généralisés dérivés à partir du modèle probit ordonné pour la fréquence des grèves et dont la forme générale est :

$$
\lambda_{i j}=\frac{\phi\left(\mu_{j-1}-\mathbf{W}_{i} \theta\right)-\phi\left(\mu_{j}-\mathbf{W}_{i} \theta\right)}{\Phi\left(\mu_{j}-\mathbf{W}_{i} \theta\right)-\Phi\left(\mu_{j-1}-\mathbf{W}_{i} \theta\right)}
$$

où $\phi$ est la fonction de densité de probabilité de la loi normale standard. Les résidus généralisés associés aux fréquences minimale et maximale des grèves $(j=0,3)$ sont calculés ainsi :

$$
\begin{gathered}
\lambda_{i 0}=-\frac{\phi\left(\mu_{1}-\mathbf{W}_{i} \theta\right)}{\Phi\left(\mu_{1}-\mathbf{W}_{i} \theta\right)} \\
\lambda_{i 3}=\frac{\phi\left(\mu_{3}-\mathbf{W}_{i} \theta\right)}{1-\Phi\left(\mu_{3}-\mathbf{W}_{i} \theta\right)}
\end{gathered}
$$

Le coefficient $\sigma_{\alpha}$ associé aux résidus généralisés, dans l'équation (5), capture la corrélation entre les termes d'erreur $\nu_{i}$ et $u_{i}$, et donc implicitement la corrélation entre les variables de fréquence des grèves $S_{i j}$ et le terme d'erreur $u_{i}$. Le coefficient $\sigma_{\alpha}$ apporte ainsi un test direct de l'endogénéité de la fréquence des grèves dans l'équation de productivité du travail. $\mathrm{Si}$ ce coefficient est statistiquement différent de 0, les variables binaires de fréquence des grèves étaient, avant l'introduction des résidus généralisés, significativement corrélées avec le terme d'erreur $u_{i}$, donc endogènes dans l'équation de productivité du travail. Sinon, il n'y a pas de biais d'hétérogénéité inobservée. Les estimations par MCO de l'équation (5) 
TABLE 3 - Equation de fréquence des grèves (probit ordonné) - première étape

\begin{tabular}{|c|c|c|c|c|c|}
\hline \multirow{3}{*}{ Variable dépendante } & \multicolumn{5}{|c|}{ Fréquence des grèves : $S^{*}$} \\
\hline & \multirow[b]{2}{*}{ Coefficients } & \multicolumn{4}{|c|}{ Effets marginaux } \\
\hline & & 0 & $1-2$ & $3-5$ & $>5$ \\
\hline $\ln K / L$ & $\begin{array}{l}0.091^{* * *} \\
(0.027)\end{array}$ & $\begin{array}{l}-0.021^{* * *} \\
(0.006)\end{array}$ & $\begin{array}{l}0.008^{* * *} \\
(0.002)\end{array}$ & $\begin{array}{l}0.005^{* * *} \\
(0.002)\end{array}$ & $\begin{array}{l}0.008^{* * *} \\
(0.002)\end{array}$ \\
\hline $\ln L$ & $\begin{array}{l}0.238^{* * *} \\
(0.025)\end{array}$ & $\begin{array}{l}-0.054^{* * *} \\
(0.005)\end{array}$ & $\begin{array}{l}0.021^{* * *} \\
(0.002)\end{array}$ & $\begin{array}{l}0.013^{* * *} \\
(0.002)\end{array}$ & $\begin{array}{l}0.021 * * * \\
(0.002)\end{array}$ \\
\hline Multi-établissements & $\begin{array}{l}-0.215^{* * *} \\
(0.082)\end{array}$ & $\begin{array}{l}0.049^{* * *} \\
(0.019)\end{array}$ & $\begin{array}{l}-0.019^{* *} \\
(0.007)\end{array}$ & $\begin{array}{l}-0.012^{* *} \\
(0.004)\end{array}$ & $\begin{array}{l}-0.019^{* * *} \\
(0.007)\end{array}$ \\
\hline Bourse & $\begin{array}{l}-0.074 \\
(0.068)\end{array}$ & $\begin{array}{l}0.017 \\
(0.015)\end{array}$ & $\begin{array}{l}-0.006 \\
(0.006)\end{array}$ & $\begin{array}{l}-0.004 \\
(0.004)\end{array}$ & $\begin{array}{l}-0.006 \\
(0.006)\end{array}$ \\
\hline$\%$ femmes & $\begin{array}{l}-0.651^{* * *} \\
(0.197)\end{array}$ & $\begin{array}{l}0.148^{* * *} \\
(0.044)\end{array}$ & $\begin{array}{l}-0.056^{* * *} \\
(0.017)\end{array}$ & $\begin{array}{l}-0.035^{* * *} \\
(0.011)\end{array}$ & $\begin{array}{l}-0.056^{* * *} \\
(0.017)\end{array}$ \\
\hline$\% \mathrm{CDD}$ & $\begin{array}{l}-0.397 \\
(0.286)\end{array}$ & $\begin{array}{l}0.090 \\
(0.065)\end{array}$ & $\begin{array}{l}-0.034 \\
(0.025)\end{array}$ & $\begin{array}{l}-0.021 \\
(0.015)\end{array}$ & $\begin{array}{l}-0.034 \\
(0.025)\end{array}$ \\
\hline \multicolumn{6}{|l|}{ CSP (ref : \% employés) } \\
\hline$\%$ cadres & $\begin{array}{l}0.841^{* * *} \\
(0.248)\end{array}$ & $\begin{array}{l}-0.191^{* * *} \\
(0.056)\end{array}$ & $\begin{array}{l}0.073^{* * *} \\
(0.021)\end{array}$ & $\begin{array}{l}0.045^{* * *} \\
(0.014)\end{array}$ & $\begin{array}{l}0.072^{* * *} \\
(0.022)\end{array}$ \\
\hline$\%$ TAM & $\begin{array}{l}1.511^{* * *} \\
(0.287)\end{array}$ & $\begin{array}{l}-0.343^{* * *} \\
(0.064)\end{array}$ & $\begin{array}{l}0.131^{* * *} \\
(0.025)\end{array}$ & $\begin{array}{l}0.081^{* * *} \\
(0.016)\end{array}$ & $\begin{array}{l}0.130^{* * *} \\
(0.026)\end{array}$ \\
\hline$\%$ ouvriers & $\begin{array}{l}0.995^{* * *} \\
(0.229)\end{array}$ & $\begin{array}{l}-0.226^{* * *} \\
(0.052)\end{array}$ & $\begin{array}{l}0.086^{* * *} \\
(0.020)\end{array}$ & $\begin{array}{l}0.054^{* * *} \\
(0.013)\end{array}$ & $\begin{array}{l}0.086^{* * *} \\
(0.020)\end{array}$ \\
\hline Présence syndicale & $\begin{array}{l}0.934^{* * *} \\
(0.169)\end{array}$ & $\begin{array}{l}-0.212^{* * *} \\
(0.038)\end{array}$ & $\begin{array}{l}0.081^{* * *} \\
(0.014)\end{array}$ & $\begin{array}{l}0.050^{* * *} \\
(0.010)\end{array}$ & $\begin{array}{l}0.080^{* * *} \\
(0.015)\end{array}$ \\
\hline Secteur (16) & $X$ & $X$ & $X$ & $X$ & $X$ \\
\hline Age entreprise (6) & $X$ & $X$ & $X$ & $X$ & $X$ \\
\hline \multicolumn{6}{|l|}{ Instruments : } \\
\hline Taux de syndicalisation $>5 \%$ & $\begin{array}{l}0.438^{* * *} \\
(0.068)\end{array}$ & $\begin{array}{l}-0.099^{* * *} \\
(0.015)\end{array}$ & $\begin{array}{l}0.038^{* * *} \\
(0.006)\end{array}$ & $\begin{array}{l}0.024 * * * \\
(0.004)\end{array}$ & $\begin{array}{l}0.038^{* * *} \\
(0.006)\end{array}$ \\
\hline Faible activité syndicale (bassin d'emploi) & $\begin{array}{l}-0.265^{* * *} \\
(0.064)\end{array}$ & $\begin{array}{l}0.060^{* * *} \\
(0.014)\end{array}$ & $\begin{array}{l}-0.023^{* * *} \\
(0.005)\end{array}$ & $\begin{array}{l}-0.014^{* * *} \\
(0.004)\end{array}$ & $\begin{array}{l}-0.023^{* * *} \\
(0.005)\end{array}$ \\
\hline$\mu_{1}$ & & & $\begin{array}{c}4.304^{* * *} \\
(0.419)\end{array}$ & & \\
\hline$\mu_{2}$ & & & $\begin{array}{c}5.046^{* * *} \\
(0.422)\end{array}$ & & \\
\hline$\mu_{3}$ & & & $\begin{array}{c}5.527^{* * *} \\
(0.424)\end{array}$ & & \\
\hline Observations & & & 2149 & & \\
\hline Log - vraisemblance & & & -1354.445 & & \\
\hline
\end{tabular}

sont consistantes mais, à moins que $\sigma_{\alpha}=0$, les écarts-type sont incorrects compte tenu de la présence de régresseurs générés. L'utilisation de la méthode bootstrap est ici utilisée pour ajuster les écarts-type.

L'existence d'un biais dû à l'hétérogénéité des effets des fréquences de grèves sur la productivité du travail peut être testée en augmentant l'équation (5) avec les termes d'interaction entre les fréquences de grèves $S_{j}$ et les résidus généralisés $\lambda_{j}$ : 


$$
\ln y_{i}=\mathbf{X}_{i}^{\prime} \beta+\sum_{j=1}^{3} \delta_{j} S_{i j}+\sigma_{\alpha} \sum_{j=0}^{3} S_{i j} \cdot \lambda_{i j}+\sum_{j=1}^{3} \sigma_{\delta_{j}} S_{i j} \cdot \lambda_{i j}+u_{i}
$$

Spécifiquement, il n'y a pas de biais d'hétérogénéité de l'effet des fréquences de grèves si les paramètres $\sigma_{\delta_{j}}$, qui capturent la corrélation entre la composante idiosyncratique de l'effet des grèves sur la productivité du travail et le terme d'erreur $\nu$, sont conjointement non-significativement différents de 0.

Les estimations des paramètres de l'équation de productivité du travail, réalisées en deuxième étape, sont reportées dans la Table 4. Les colonnes (1) et (2) intègrent les coefficients obtenus respectivement sans et avec introduction des résidus généralisés. Les fréquences des grèves sont considérées comme exogènes dans la spécification (1) et l'équation de productivité du travail est estimée par la méthode des MCO, en contrôlant la robustesse des écarts-type à la présence d'hétéroscédasticité. Dans la spécification (2), le coefficient $\sigma_{\alpha}$ est statistiquement différent de 0 ; nous pouvons donc rejeter l'hypothèse de corrélation nulle entre les fréquences de grèves $S_{j}$ et le terme d'erreur $u$. Il y a bien un problème d'endogénéité entre productivité du travail et fréquence des grèves, introduisant un biais dans les estimations (1). A la différence du traitement binaire, l'interprétation du coefficient associé aux résidus généralisés n'est pas aisée dans le cadre de traitements multiples. Ce coefficient peut, en effet, indiquer une corrélation non-nulle entre le résidu $u$ et une fréquence particulière de grèves, par rapport à l'ensemble des autres fréquences.

La non-prise en compte de ce biais d'hétérogénéité inobservée conduit à sous-estimer l'effet positif des fréquences de grèves $S_{1}$ et $S_{2}$ sur la productivité du travail. Les coefficients positifs associés à $S_{1}$ et $S_{2}$ augmentent sensiblement avec l'introduction des résidus généralisés $\lambda_{j}$. Nous obtenons ainsi que les entreprises ayant connu 1 à 2 grèves, entre 2002 et 2004, présentent un niveau de productivité du travail significativement plus élevé que les entreprises non-grévistes, en 2004 - d'environ 12\%. Une fréquence de 3 à 5 grèves entraîne une hausse significative de la productivité du travail, qui s'avère être davantage prononcée en corrigeant le biais d'hétérogénéité inobservée - environ 29\% par rapport aux entreprises non-grévistes. L'occurrence de plus de 5 grèves, quant à elle, n'est pas associée à une différence significative de productivité du travail, bien que son coefficient $\left(S_{3}\right)$ devienne positif en tenant compte de l'hétérogénéité inobservée.

Dans la spécification (3), Table 4, nous contrôlons le biais potentiel d'hétérogénéité dans les paramètres $\delta_{j}$, en augmentant l'équation de productivité du travail avec les termes d'interaction entre les fréquences de grèves $S_{j}$ et les résidus généralisés $\lambda_{j}$. En menant un test de $\chi^{2}$ sur les coefficients de ces termes d'interaction, nous pouvons rejeter l'hypothèse que les $\sigma_{\delta_{j}}$ sont conjointement non-différents de 0 . Il y a donc bien hétérogénéité de l'effet des fréquences de grèves $S_{j}$ sur la productivité en fonction de caractéristiques inobservables. Toutefois, seul le coefficient du terme d'interaction $S_{3} \times \lambda$ est statistiquement différent de 0. L'effet de cette fréquence de grèves est donc particulièrement susceptible de varier en fonction des caractéristiques inobservables. L'intervalle des valeurs est sensiblement plus important pour cette fréquence des grèves et peut expliquer en partie le niveau de significativité du coefficient $\sigma_{\lambda_{3}}$. Cette significativité nous amène à considérer l'effet de $S_{3}$ conditionnellement aux caractéristiques inobservables. Cet effet conditionnel s'obtient en dérivant l'équation (9) par rapport à $S_{3}$, soit : $\delta_{3}+\sigma_{\delta_{3}} \lambda$, ce qui équivaut ici à $-0.158+0.315 \lambda$. Toutefois, suite à un 
TABLE 4 - Equations de productivité du travail - deuxième étape

\begin{tabular}{|c|c|c|c|c|c|c|}
\hline \multirow{3}{*}{ Variable dépendante } & \multicolumn{6}{|c|}{ Productivité du travail $: \ln y$} \\
\hline & \multicolumn{2}{|c|}{ (1) } & \multicolumn{2}{|c|}{$(2)$} & \multicolumn{2}{|c|}{$(3)$} \\
\hline & Coef. & (E.-T.) & Coef. & (E.-T.) & Coef. & (E.-T.) \\
\hline$S_{1}: 1-2$ grèves & 0.013 & $(0.028)$ & $0.124^{*}$ & $(0.066)$ & $0.187^{* * *}$ & $(0.069)$ \\
\hline$S_{2}: 3-5$ grèves & $0.141^{* * *}$ & $(0.049)$ & $0.289^{* * *}$ & $(0.097)$ & $0.264^{* *}$ & $(0.123)$ \\
\hline$S_{3}:>5$ grèves & -0.054 & $(0.062)$ & 0.141 & $(0.112)$ & -0.158 & $(0.209)$ \\
\hline $\ln K / L$ & $0.146^{* * *}$ & $(0.011)$ & $0.141^{* * *}$ & $(0.011)$ & $0.142^{* * *}$ & $(0.011)$ \\
\hline $\ln L$ & $0.023^{* * *}$ & $(0.008)$ & $0.014^{*}$ & $(0.008)$ & 0.014 & $(0.009)$ \\
\hline Multi-établissements & 0.004 & $(0.025)$ & 0.014 & $(0.026)$ & 0.012 & $(0.025)$ \\
\hline Bourse & $0.109 * * *$ & $(0.024)$ & $0.111^{* * *}$ & $(0.024)$ & $0.102^{* * *}$ & $(0.024)$ \\
\hline$\%$ femmes & $-0.174^{* * *}$ & $(0.054)$ & $-0.151^{* * *}$ & $(0.054)$ & $-0.134^{* *}$ & $(0.053)$ \\
\hline$\% \mathrm{CDD}$ & 0.107 & $(0.074)$ & 0.119 & $(0.075)$ & $0.124^{*}$ & $(0.073)$ \\
\hline \multicolumn{7}{|c|}{ CSP (ref : \% employés) } \\
\hline$\%$ cadres & $0.916^{* * *}$ & $(0.079)$ & $0.901^{* * *}$ & $(0.080)$ & $0.896^{* * *}$ & $(0.081)$ \\
\hline$\%$ TAM & $0.449^{* * *}$ & $(0.083)$ & $0.399^{* * *}$ & $(0.085)$ & $0.392^{* * *}$ & $(0.086)$ \\
\hline$\%$ ouvriers & $-0.201^{* * *}$ & $(0.057)$ & $-0.223^{* * *}$ & $(0.059)$ & $-0.235^{* * *}$ & $(0.057)$ \\
\hline Présence syndicale & $-0.119 * * *$ & $(0.028)$ & $-0.133^{* * *}$ & $(0.029)$ & $-0.157 * * *$ & $(0.032)$ \\
\hline$\lambda$ & - & & $-0.084^{* *}$ & $(0.042)$ & $-0.152^{* *}$ & $(0.070)$ \\
\hline$S_{1} \times \lambda$ & - & & - & & 0.027 & $(0.070)$ \\
\hline$S_{2} \times \lambda$ & - & & - & & 0.123 & $(0.103)$ \\
\hline$S_{3} \times \lambda$ & - & & - & & $0.315^{* *}$ & $(0.123)$ \\
\hline$\chi^{2}(3)$ & & & & & $7.29^{*}$ & 0.063 \\
\hline $\begin{array}{l}\text { Secteur (16) } \\
\text { Age entreprise (6) }\end{array}$ & \multicolumn{2}{|c|}{$\begin{array}{l}X \\
X\end{array}$} & \multicolumn{2}{|c|}{$\begin{array}{l}X \\
X\end{array}$} & \multicolumn{2}{|r|}{$\begin{array}{l}X \\
X\end{array}$} \\
\hline Constante & $3.803^{* * *}$ & $(0.132)$ & $3.874^{* * *}$ & $(0.141)$ & $3.862^{* * *}$ & $(0.136)$ \\
\hline \multicolumn{7}{|c|}{ Test de Wald sur instruments } \\
\hline Equation $S^{*}$ & - & & 60.57 & $(0.000)$ & 60.57 & $(0.000)$ \\
\hline Equation $\ln y$ & - & & 0.01 & $(0.988)$ & 0.01 & $(0.988)$ \\
\hline Observations & 2149 & & 2149 & & 2149 & \\
\hline$R^{2}$ & 0.515 & & 0.509 & & 0.511 & \\
\hline \multicolumn{7}{|c|}{$\begin{array}{l}\text { NOTES }{ }^{* * *} \text { significatif à } 1 \%,{ }^{* *} \text { à } 5 \%,{ }^{*} \text { à } 10 \% \text {. Les écarts-type, entre parenthèses, sont robustes à la présence } \\
\text { d'hétéroscédasticité, ajustés par la méthode bootstrap ( } 1000 \text { réplications) dans }(2) \text { et }(3) \text {. } \lambda \text { correspond au } \\
\text { résidu généralisé, obtenu à partir du modèle probit ordonné pour la fréquence des grèves, en première étape. } \\
\text { Instruments : taux de syndicalisation de la main d'oeuvre supérieur à } 5 \% \text { (binaire), activité syndicale peu intense } \\
\text { dans le bassin d'emploi (binaire). TAM = Techniciens et Agents de Maitrise. }\end{array}$} \\
\hline
\end{tabular}

test de Wald sur cette combinaison linéaire $(p=0.320)$, nous ne pouvons rejeter l'hypothèse nulle de significativité de cet effet conditionnel. Ainsi, en dépit du biais qu'ils permettent de corriger, l'introduction de ces termes d'interaction ne modifie pas la configuration des résultats pour les variables d'intérêt.

La prise en compte de ces biais liés à l'hétérogénéité inobservée entraîne toutefois des changements significatifs dans les coefficients de plusieurs variables de contrôle. Notamment, l'effet positif de la taille de l'entreprise sur la productivité du travail tend à disparaître en contrôlant ces biais statistiques tandis que la part des salariés en CDD est désormais associée à un effet positif, quoique faiblement significatif. Ce dernier effet est tout à fait cohérent avec la littérature existante, à l'instar des effets positifs associés à l'intensité capitalistique, à la 
cotation en bourse et à la part des travailleurs qualifiés dans la main d'oeuvre. De même, le taux de féminisation de la main d'oeuvre apparaît affecter négativement la productivité du travail, un effet largement documenté dans la littérature (e.g. Zwick 2004). Au regard de l'effet de la présence syndicale sur la productivité, la littérature n'offre pas de consensus sur son sens. En isolant l'effet des grèves, un des canaux reconnus de l'effet syndical, nous estimons que la présence syndicale affecte négativement la productivité du travail. Ce dernier effet renvoie, dans la littérature, au visage de monopole du syndicalisme (e.g. Freeman et Medoff 1984), associé notamment à une limitation du pouvoir discrétionnaire de l'employeur et à la mise en oeuvre de pratiques de travail restrictives.

Ces premières estimations mettent en évidence un effet non-linéaire de la fréquence des grèves sur la productivité du travail : un effet positif et croissant jusqu'à 5 grèves puis neutre au-delà de ce seuil. Cet effet non-linéaire s'apparente fortement à l'effet présenté par Knight (1989), dans son étude des industries manufacturières britanniques. Cet effet ne peut se concevoir comme un effet direct, de court terme, mais doit plutôt s'interpréter comme un effet indirect, à plus long terme, compte tenu de l'information dont nous disposons sur la fréquence des grèves. A l'instar de Knight (1989), nos résultats ne mettent pas en évidence un effet indirect négatif des grèves sur la productivité du travail, qui pourrait relever des expressions individuelles de conflit qui leur sont associées (e.g. Flaherty 1987 ; Kleiner et al. 2002). L'effet positif d'une fréquence modérée des grèves que nous estimons corrobore davantage la vision des auteurs de l'école d'Harvard, pour qui la grève exercerait un rôle d'expression collective (e.g. Freeman et Medoff 1984; Godard 1992), positif pour la productivité des entreprises. À une faible fréquence, les grèves semblent ainsi avoir un rôle 'thérapeutique' (Knight 1989, p. 370) dans les entreprises. Au-delà de 5 grèves sur la période considérée, ce rôle positif des grèves ne semble plus être à l'oeuvre ou sinon compensé par un effet contradictoire, propre à une telle fréquence des grèves. En effet, une forte fréquence des grèves peut être source de fortes perturbations dans la production (e.g. Flaherty 1987) mais également être associée à une plus forte expression individuelle de conflit des salariés, toutes néfastes pour la productivité.

\section{Variation de l'effet des grèves selon l'absentéisme}

Nous prolongeons ici notre analyse empirique, en évaluant si et dans quelle mesure l'effet de la fréquence des grèves varie en fonction de l'existence d'un problème d'absentéisme dans l'entreprise. Nous estimons désormais deux équations de productivité du travail, pour chaque sous-échantillon sélectionné en fonction de notre variable binaire d'absentéisme $(A=0,1)$ :

$$
\ln y_{k i}=\mathbf{X}_{i}^{\prime} \beta_{k}+\sum_{j=1}^{3} \delta_{k j} S_{i j}+u_{k i} ; \quad k=0,1
$$

où $\ln y_{0 i}$ et $\ln y_{1 i}$ représentent, respectivement, le logarithme de la productivité du travail de l'entreprise $i$ parmi les entreprises sans problème d'absentéisme $(A=0)$ et avec un problème d'absentéisme $(A=1)$. Le vecteur $\mathbf{X}$ est le même que précédemment. Les $u_{k}$ sont des termes d'erreur supposés être normalement distribués, de moyenne nulle et de variance $\sigma_{k}$. Outre le problème de corrélation non-nulle entre les facteurs inobservés déterminant la fréquence des grèves et le niveau de productivité du travail, l'estimation de ces deux équations est compliquée par le fait que la sélection de ces sous-échantillons peut ne pas être totalement 
exogène, même en contrôlant un ensemble de caractéristiques observées. Des caractéristiques inobservables peuvent affecter à la fois la probabilité qu'il y ait un problème d'absentéisme et le niveau de productivité du travail dans l'entreprise. Pour tenir compte de ce biais potentiel, nous augmentons le modèle initial avec une équation pour la probabilité d'absentéisme dans l'entreprise, en première étape :

$$
A^{*}=\sum_{j=1}^{3} \zeta_{j} S_{i j}+\mathbf{V}_{i}^{\prime} \gamma+\eta_{i}, \quad A=1\left(A^{*}>0\right), \quad \eta_{i} \sim N(0,1)
$$

où $A^{*}$ est la variable latente d'absentéisme dans l'entreprise, $\mathbf{V}$ est un vecteur de caractéristiques exogènes qui intègre l'ensemble des variables $\mathbf{X}$ introduites dans l'équation de productivité du travail ln y ainsi que les instruments $\mathbf{Z}$, précédemment introduits dans l'équation de fréquence des grèves; $\eta$ capture les facteurs inobservés qui influencent la probabilité que l'entreprise rencontre un problème d'absentéisme et est supposé être normalement distribué. Une difficulté supplémentaire dans cette spécification est que les variables binaires $j$ de fréquence des grèves peuvent être endogènes à l'existence d'un problème d'absentéisme.

L'endogénéité et l'autosélection sont modélisées en permettant une corrélation entre les termes d'erreur $u_{0}, u_{1}, \nu$ et $\eta$. Spécifiquement : $\sigma_{k \nu}=\operatorname{cov}\left(u_{k}, \nu\right), \sigma_{k \eta}=\operatorname{cov}\left(u_{k}, \eta\right)$ où $k=0,1$; $\rho=\operatorname{corr}(\nu, \eta)$. Si les équations de productivité du travail $\left(\ln y_{k}\right)$, de fréquence des grèves $\left(S^{*}\right)$ et d'absentéisme $\left(A^{*}\right)$ sont corrélées à travers les inobservables, les espérances conditionnelles des termes d'erreur $u_{k i}$ dans (10) sont différentes de 0 et les estimations MCO ne sont donc pas consistantes. Nous utilisons un modèle en deux étapes, où les termes de correction introduits dans les équations de productivité du travail contrôlent simultanément les deux formes d'endogénéité, impliquant des distributions normales bivariées. En première étape, nous estimons simultanément les paramètres des équations d'absentéisme et de fréquence des grèves par maximum de vraisemblance, incluant le coefficient de corrélation $\rho^{12}$. Si $\rho$ est significativement différent de 0 , cela implique que la fréquence des grèves est endogène avec l'existence d'un problème d'absentéisme.

Pour permettre l'identification du modèle estimé en première étape, nous introduisons dans l'équation de fréquence des grèves $S^{*}$ des variables instrumentales supplémentaires, exclues à juste titre des équations d'absentéisme et de productivité du travail. Les instruments ici retenus portent sur l'identité des syndicats présents dans l'entreprise. Des études françaises soulignent que la probabilité d'occurrence de grèves augmente lorsque certains syndicats sont représentés dans l'entreprise (e.g. Béroud et al. 2008), au premier rang desquels figurent notamment la Confédération Générale du Travail (CGT) et la Confédération Française Démocratique du Travail (CFDT). Deux variables binaires, indiquant chacune la présence d'un de ces deux syndicats dans l'entreprise, sont donc retenues comme instruments additionnels dans l'équation de fréquence des grèves $S^{*}$. Les statistiques des tests de Wald menés sur les paramètres de ces variables dans les équations d'absentéisme et de productivité du travail sont reportées respectivement au bas des Tables 5 et 6 .

Nous reportons, dans la Table 5 , les coefficients estimés des équations d'absentéisme $A^{*}$ et de fréquence des grèves $S^{*}$, lorsque estimées séparément (1) et simultanément (2). Dans

12. Le modèle estimé en première étape est proche du modèle probit bivarié, en tenant compte cependant de la nature ordinale de la fréquence des grèves. L'estimation du modèle est réalisée grâce à la commande cmp (voir Roodman 2011), sous Stata. 
TABLE 5 - Equations d'absentéisme (probit) et de fréquence des grèves (probit ordonné) Première étape

\begin{tabular}{|c|c|c|c|c|c|c|c|c|}
\hline \multirow{3}{*}{ Variable dépendante } & \multicolumn{4}{|c|}{$\begin{array}{l}\text { (1) Fréquence de grèves exogène } \\
\text { Equations indépendantes }\end{array}$} & \multicolumn{4}{|c|}{$\begin{array}{l}\text { (2) Fréquence de grèves endogène } \\
\text { Equations simultanées }\end{array}$} \\
\hline & \multicolumn{2}{|c|}{ Absentéisme $A^{*}$} & \multicolumn{2}{|c|}{ Fréq. grèves $S^{*}$} & \multicolumn{2}{|c|}{ Absentéisme $A^{*}$} & \multicolumn{2}{|c|}{ Fréq. grèves $S^{*}$} \\
\hline & Coef. & (E.-T.) & Coef. & (E.-T.) & Coef. & (E.-T.) & Coef. & (E.-T.) \\
\hline$S_{1}: 1-2$ grèves & 0.104 & $(0.088)$ & - & & -0.423 & $(0.259)$ & - & \\
\hline$S_{2}: 3-5$ grèves & 0.064 & $(0.137)$ & - & & $-0.635^{*}$ & $(0.350)$ & - & \\
\hline$S_{3}:>5$ grèves & $0.270^{*}$ & $(0.139)$ & - & & -0.673 & $(0.456)$ & - & \\
\hline $\ln K / L$ & -0.037 & $(0.024)$ & $0.086^{* * *}$ & $(0.028)$ & -0.014 & $(0.026)$ & $0.085^{* * *}$ & $(0.027)$ \\
\hline $\ln L$ & -0.000 & $(0.022)$ & $0.204^{* * *}$ & $(0.027)$ & 0.039 & $(0.029)$ & $0.201^{* * *}$ & $(0.026)$ \\
\hline Multi-établissements & -0.109 & $(0.072)$ & $-0.230 * * *$ & $(0.082)$ & $-0.140 *$ & $(0.072)$ & $-0.221 * * *$ & $(0.081)$ \\
\hline Bourse & -0.063 & $(0.065)$ & -0.108 & $(0.068)$ & -0.077 & $(0.064)$ & -0.102 & $(0.068)$ \\
\hline$\%$ femmes & $0.536^{* * *}$ & $(0.162)$ & $-0.660 * * *$ & $(0.200)$ & $0.437^{* * *}$ & $(0.168)$ & $-0.731^{* * *}$ & $(0.203)$ \\
\hline$\% \mathrm{CDD}$ & -0.243 & $(0.262)$ & $-0.487^{*}$ & $(0.290)$ & -0.282 & $(0.261)$ & -0.382 & $(0.295)$ \\
\hline \multicolumn{9}{|l|}{ CSP (ref : \% employés) } \\
\hline$\%$ cadres & $-1.356^{* * *}$ & $(0.210)$ & $0.796^{* * *}$ & $(0.250)$ & $-1.217^{* * *}$ & $(0.224)$ & $0.766^{* * *}$ & $(0.248)$ \\
\hline$\%$ TAM & $-0.665^{* * *}$ & $(0.230)$ & $1.469^{* * *}$ & $(0.292)$ & $-0.429 *$ & $(0.255)$ & $1.476^{* * *}$ & $(0.286)$ \\
\hline$\%$ ouvriers & $0.467 * * *$ & $(0.167)$ & $0.983^{* * *}$ & $(0.229)$ & $0.549^{* * *}$ & $(0.168)$ & $0.956^{* * *}$ & $(0.227)$ \\
\hline Présence syndicale & $0.332^{* * *}$ & $(0.091)$ & $0.514^{* * *}$ & $(0.188)$ & $0.354^{* * *}$ & $(0.090)$ & $0.544^{* * *}$ & $(0.187)$ \\
\hline \multicolumn{9}{|l|}{ Instruments : } \\
\hline Syndicalisation > $>5 \%$ & $0.230^{* * *}$ & $(0.065)$ & $0.417^{* * *}$ & $(0.069)$ & $0.308^{* * *}$ & $(0.072)$ & $0.427^{* * *}$ & $(0.069)$ \\
\hline $\begin{array}{l}\text { Faible activité syndicale } \\
\text { (bassin d'emploi) }\end{array}$ & $-0.185^{* * *}$ & $(0.059)$ & $-0.257^{* * *}$ & $(0.065)$ & $-0.234^{* * *}$ & $(0.061)$ & $-0.253^{* * *}$ & $(0.064)$ \\
\hline $\mathrm{CFDT}^{*}$ & - & & $0.200^{* *}$ & $(0.090)$ & - & & $0.184^{* *}$ & $(0.089)$ \\
\hline $\mathrm{CGT}^{*}$ & - & & $0.474^{* * *}$ & $(0.098)$ & - & & $0.459^{* * *}$ & $(0.098)$ \\
\hline Secteur (16) & \multicolumn{2}{|c|}{$X$} & \multicolumn{2}{|c|}{$X$} & \multicolumn{2}{|c|}{$X$} & \multicolumn{2}{|c|}{$X$} \\
\hline Age entreprise (6) & \multicolumn{2}{|c|}{$X$} & \multicolumn{2}{|c|}{$X$} & \multicolumn{2}{|c|}{$X$} & \multicolumn{2}{|c|}{$X$} \\
\hline$\rho$ & & & & & $0.408^{* *}$ & $(0.203)$ & & \\
\hline Constante & 0.267 & $(0.306)$ & & & -0.066 & $(0.348)$ & & \\
\hline$\mu_{1}$ & & & $4.049^{* * *}$ & $(0.417)$ & & & $4.066^{* * *}$ & $(0.422)$ \\
\hline$\mu_{2}$ & & & $4.806^{* * *}$ & $(0.419)$ & & & $4.820^{* * *}$ & $(0.425)$ \\
\hline$\mu_{3}$ & & & $5.291 * * *$ & $(0.421)$ & & & $5.304^{* * *}$ & $(0.427)$ \\
\hline $\begin{array}{l}\text { Test de Wald } \\
\text { sur instruments* }\end{array}$ & 0.52 & $(0.771)$ & 31.09 & $(0.000)$ & & & & \\
\hline Observations & 214 & & 214 & & & & & \\
\hline Log-vraisemblance & -1323 & 763 & -1337 & 421 & & & .000 & \\
\hline
\end{tabular}

NOTES : ${ }^{* * *}$ significatif à $1 \%,{ }^{* *}$ à $5 \%,{ }^{*}$ à $10 \%$. Les écarts-type, entre parenthèses, sont robustes à la présence d'hétéroscédasticité. TAM = Techniciens et Agents de Maîtrise.

le modèle d'équations simultanées, le coefficient $\rho$ estimé est significativement différent de 0 , indiquant que les termes d'erreur $\nu$ et $\eta$ sont corrélés; la fréquence des grèves est donc endogène à l'existence d'un problème d'absentéisme dans l'entreprise. Le fait de contrôler l'endogénéité des variables de fréquence de grèves dans l'équation d'absentéisme modifie sensiblement les paramètres estimés pour ces variables. Toutefois, en raison de la forme réduite des équations $A^{*}$ et $S^{*}$, il convient d'interpréter les coefficients de ces deux équations avec précaution. Compte tenu de l'endogénéité des fréquences de grèves $S_{j}$ dans l'équation d'absentéisme $A^{*}$, la méthode d'estimation décrite ci-dessus sera préférée à une correction 
séparée ${ }^{13}$ de l'auto-sélection et de l'endogénéité de $S^{*}$ dans l'équation de productivité du travail, en deuxième étape.

Les paramètres estimés lors de la première étape sont utilisés pour calculer les termes de correction $\lambda_{1}$ et $\lambda_{2}$, ensuite introduits dans les équations de productivité du travail, en deuxième étape :

$$
\ln y_{k i}=\mathbf{X}_{i}^{\prime} \beta_{k}+\sum_{j=1}^{3} \delta_{k j} S_{i j}+\sigma_{k \nu} \lambda_{1 k i}+\sigma_{k \eta} \lambda_{2 k i}+u_{k i} ; \quad k=0,1
$$

Nous détaillons la procédure permettant d'obtenir les termes de correction $\lambda_{1}$ et $\lambda_{2}$ dans une section annexe, à la fin de l'article. Une fois l'endogénéité entre la fréquence de grèves et l'absentéisme contrôlée, $\lambda_{1}$ et $\lambda_{2}$ contrôlent pour la sélection non-aléatoire des entreprises dans l'état $k=0,1$ et pour l'endogénéité des fréquences de grèves dans l'équation de productivité du travail. Le niveau de significativité des paramètres $\sigma_{k \nu}$ et $\sigma_{k \eta}$ indiquent respectivement l'endogénéité de la fréquence des grèves et l'auto-sélection dans l'état $k=0,1$. Si $\sigma_{k \nu} \neq 0$ et/ou $\sigma_{k \eta} \neq 0$, les écarts-type estimés sont biaisés du fait de la présence de régresseurs générés et nécessitent d'être ajustés, par la méthode bootstrap.

Les estimations issues de cette deuxième étape sont reportées dans la Table 6. Les estimations reportées dans la partie (1) sont issues de modèles exogènes, ignorant les biais d'endogénéité des variables de fréquence des grèves et de sélection endogène des sous-échantillons. Ces biais sont contrôlés dans la partie (2) grâce à l'introduction des termes de correction $\lambda_{1}$ et $\lambda_{2}$, présentés ci-dessus. Les coefficients associés à $\lambda_{\eta}$ nous indiquent que les estimations reportées dans (1) souffrent d'un problème d'endogénéité, dans le seul cas des entreprises non-concernées par un problème d'absentéisme $(A=0)$; dans le cas des entreprises concernées par un problème d'absentéisme $(A=1)$, le paramètre $\lambda_{\eta}$ n'a pas d'effet statistiquement significatif. Par contre, la sélection non-aléatoire des deux sous-échantillons ne semble pas causer de biais significatif dans les estimations, compte tenu de la non-significativité du terme de correction $\lambda_{\eta}$ dans chaque sous-échantillon ${ }^{14}$. L'absence de prise en compte de l'endogénéité de la fréquence des grèves, lorsque $A=0$, mène à une sous-estimation de l'effet positif de chaque $S_{j}$ sur la productivité du travail ln $y$. Une fois contrôlé ce biais, nous obtenons un effet significativement positif du nombre de grèves sur la productivité du travail, croissant jusqu'à 5 grèves, puis décroissant au-delà. A l'inverse, en présence d'un problème d'absentéisme $(A=1)$, les grèves ne présentent aucun effet significatif sur la productivité du travail.

Nous testons enfin, dans la partie (3), l'existence d'un biais d'hétérogénéité de l'effet des fréquences de grèves $S_{j}$ en fonction de caractéristiques inobservables, en introduisant les termes d'interaction $S_{j} \times \lambda_{\eta}$. Ces termes d'interaction ne présentent pas d'effet significatif sur la productivité du travail, tant séparément que conjointement, comme l'indique le test de $\chi^{2}$ reporté au bas de la Table 6 . Il apparait ainsi qu'en contrôlant l'absentéisme des salariés, par la constitution de ces deux sous-échantillons, l'effet des fréquences de grèves ne présente plus de

13. Cette méthode consiste, à l'inverse, à introduire dans l'équation de productivité du travail ln $y$ l'inverse du ratio de Mills et les résidus généralisés, calculés respectivement à partir des équations probit et probit ordonné, estimées séparément en première étape. Ces estimations ne sont pas reportées dans l'article mais sont disponibles sur demande à l'auteur.

14. Les paramètres estimés s'avèrent ainsi être très proches de ceux obtenus en ne contrôlant que l'endogénéité des fréquences de grèves $S_{j}$ dans les équations de productivité du travail ln $y_{k i}$. Ces estimations ne sont pas reportées dans l'article mais sont disponibles sur demande à l'auteur. 
TABLE 6 - Equations de productivité du travail selon $A=0,1$ - Deuxième étape

\begin{tabular}{|c|c|c|c|c|c|c|c|c|c|c|c|c|}
\hline & \multicolumn{12}{|c|}{ Productivité du travail $: \ln y$} \\
\hline & \multicolumn{4}{|c|}{$(1)$} & \multicolumn{4}{|c|}{$(2)$} & \multicolumn{4}{|c|}{ (3) } \\
\hline & \multicolumn{2}{|c|}{$A=0$} & \multicolumn{2}{|c|}{$A=1$} & \multicolumn{2}{|c|}{$A=0$} & \multicolumn{2}{|c|}{$A=1$} & \multicolumn{2}{|c|}{$A=0$} & \multicolumn{2}{|c|}{$A=1$} \\
\hline & Coef. & (E.-T.) & Coef. & (E.-T.) & Coef. & (E.-T.) & Coef. & (E.-T.) & Coef. & (E.-T.) & Coef. & (E.-T.) \\
\hline$S_{1}: 1-2$ grèves & 0.068 & $(0.048)$ & -0.022 & $(0.033)$ & $0.275^{* *}$ & $(0.108)$ & -0.045 & $(0.090)$ & $0.312^{* * *}$ & $(0.120)$ & 0.035 & $(0.110)$ \\
\hline$S_{2}: 3-5$ grèves & $0.278^{* * *}$ & $(0.083)$ & 0.053 & $(0.057)$ & $0.552^{* * *}$ & $(0.142)$ & 0.031 & $(0.122)$ & $0.664^{* * *}$ & $(0.214)$ & 0.039 & $(0.162)$ \\
\hline$S_{3}:>5$ grèves & -0.031 & $(0.097)$ & -0.069 & $(0.082)$ & $0.318^{*}$ & $(0.176)$ & -0.070 & $(0.153)$ & -0.123 & $(0.342)$ & -0.235 & $(0.263)$ \\
\hline $\ln K / L$ & $0.127 * * *$ & $(0.015)$ & $0.161^{* * *}$ & $(0.015)$ & $0.120^{* * *}$ & $(0.015)$ & $0.158^{* * *}$ & $(0.015)$ & $0.121^{* * *}$ & $(0.015)$ & $0.160 * * *$ & $(0.016)$ \\
\hline $\ln L$ & 0.016 & $(0.012)$ & $0.027^{* * *}$ & $(0.010)$ & 0.004 & $(0.013)$ & $0.022^{* *}$ & $(0.011)$ & 0.008 & $(0.014)$ & $0.023^{*}$ & $(0.012)$ \\
\hline Multi-étab. & -0.026 & $(0.040)$ & 0.043 & $(0.033)$ & -0.019 & $(0.039)$ & 0.044 & $(0.034)$ & -0.021 & $(0.043)$ & 0.042 & $(0.033)$ \\
\hline Bourse & $0.161^{* * *}$ & $(0.038)$ & $0.062^{* *}$ & $(0.030)$ & $0.165^{* * *}$ & $(0.039)$ & $0.062^{* *}$ & $(0.030)$ & $0.150^{* * *}$ & $(0.037)$ & $0.055^{*}$ & $(0.029)$ \\
\hline$\%$ femmes & -0.129 & $(0.099)$ & $-0.147^{* *}$ & $(0.064)$ & -0.088 & $(0.101)$ & $-0.129 *$ & $(0.067)$ & -0.090 & $(0.103)$ & -0.106 & $(0.069)$ \\
\hline$\%$ CDD & $0.211^{*}$ & $(0.117)$ & -0.083 & $(0.089)$ & $0.214^{*}$ & $(0.122)$ & -0.082 & $(0.095)$ & $0.206^{*}$ & $(0.122)$ & -0.077 & $(0.092)$ \\
\hline \multicolumn{13}{|c|}{ CSP (ref : \% employés) } \\
\hline$\%$ cadres & $0.880^{* * *}$ & $(0.104)$ & $1.003^{* * *}$ & $(0.132)$ & $0.846^{* * *}$ & $(0.103)$ & $0.959^{* * *}$ & $(0.141)$ & $0.847^{* * *}$ & $(0.107)$ & $0.971^{* * *}$ & $(0.137)$ \\
\hline$\%$ TAM & $0.405^{* * *}$ & $(0.120)$ & $0.474^{* * *}$ & $(0.118)$ & $0.332^{* * *}$ & $(0.119)$ & $0.435^{* * *}$ & $(0.119)$ & $0.333^{* * *}$ & $(0.124)$ & $0.436^{* * *}$ & $(0.119)$ \\
\hline$\%$ ouvriers & -0.123 & $(0.091)$ & $-0.211^{* * *}$ & $(0.075)$ & -0.136 & $(0.091)$ & $-0.219 * * *$ & $(0.077)$ & -0.130 & $(0.094)$ & $-0.236^{* * *}$ & $(0.080)$ \\
\hline Présence syndicale & $-0.131 * * *$ & $(0.041)$ & $-0.077^{*}$ & $(0.040)$ & $-0.133^{* * *}$ & $(0.041)$ & $-0.082^{*}$ & $(0.043)$ & $-0.142^{* * *}$ & $(0.045)$ & $-0.116^{* *}$ & $(0.049)$ \\
\hline$\lambda_{\nu}$ & - & & - & & $-0.144^{* *}$ & $(0.062)$ & 0.009 & $(0.054)$ & -0.179 & $(0.161)$ & -0.089 & $(0.117)$ \\
\hline$\lambda_{\eta}$ & - & & - & & -0.012 & $(0.026)$ & 0.071 & $(0.073)$ & -0.032 & $(0.050)$ & 0.034 & $(0.077)$ \\
\hline$S_{1} \times \lambda_{\nu}$ & - & & - & & - & & - & & 0.018 & $(0.181)$ & 0.068 & $(0.106)$ \\
\hline$S_{2} \times \lambda_{\nu}$ & - & & - & & - & & - & & -0.033 & $(0.202)$ & 0.172 & $(0.118)$ \\
\hline$S_{3} \times \lambda_{\nu}$ & - & & - & & - & & - & & 0.318 & $(0.262)$ & 0.287 & $(0.212)$ \\
\hline$\chi^{2}(3)$ & & & & & & & & & 2.76 & $(0.430)$ & 3.64 & $(0.303)$ \\
\hline Secteur (16) & \multicolumn{2}{|l|}{$X$} & \multicolumn{2}{|l|}{$X$} & \multicolumn{2}{|c|}{$X$} & \multicolumn{2}{|l|}{$X$} & \multicolumn{2}{|c|}{$X$} & \multicolumn{2}{|c|}{$X$} \\
\hline Age entreprise (6) & \multicolumn{2}{|c|}{$X$} & \multicolumn{2}{|c|}{$X$} & \multicolumn{2}{|c|}{$X$} & \multicolumn{2}{|c|}{$X$} & \multicolumn{2}{|c|}{$X$} & \multicolumn{2}{|l|}{$X$} \\
\hline Constante $u_{k i}$ & $4.088^{* * *}$ & $(0.219)$ & $3.473^{* * *}$ & $(0.150)$ & $4.186^{* * *}$ & $(0.232)$ & $3.524^{* * *}$ & $(0.163)$ & $4.171^{* * *}$ & $(0.226)$ & $3.500^{* * *}$ & $(0.168)$ \\
\hline $\begin{array}{l}\text { Observations } \\
R^{2}\end{array}$ & \multicolumn{2}{|c|}{1009} & \multicolumn{2}{|c|}{1140} & \multicolumn{2}{|c|}{1009} & \multicolumn{2}{|c|}{1140} & \multicolumn{2}{|c|}{1009} & \multicolumn{2}{|c|}{1140} \\
\hline
\end{tabular}

NOTES : *** significatif à 1\%, ${ }^{* *}$ à $5 \%,{ }^{*}$ à $10 \%$. Les écarts-type, entre parenthèses, sont robustes à la présence d'hétéroscédasticité, ajustés avec la méthode bootstrap (1000 réplications). $\lambda_{\nu}$ et $\lambda_{\eta}$ correspondent aux termes de correction calculés à partir du modèle bivarié pour $A^{*}$ et $S^{*} \cdot \chi^{2}(3)$ indique le résultat du test de Wald réalisé sur les termes d'interaction $S_{j} \times \lambda_{\nu}$.

biais d'hétérogénéité en fonction des caractéristiques inobservables. Ainsi, il est vraisemblable que le niveau d'absentéisme soit une caractéristique inobservable source d'hétérogénéité dans l'effet de la fréquence de grèves sur la productivité du travail.

Les résultats de ces dernières estimations permettent de vérifier empiriquement que l'estimation de l'effet de la fréquence des grèves sur la productivité du travail dépend sensiblement de la façon dont les grèves s'articulent avec l'expression individuelle de conflit des salariés dans l'entreprise, l'absentéisme dans notre cas. Dans la littérature, les auteurs n'ont, jusque là, qu'émis des hypothèses sur le rôle de cette relation entre grèves et formes individuelles de conflit quant à l'effet des grèves sur la productivité du travail (e.g. Flaherty 1987 ; Kleiner et al. 2002 ; McHugh 1991). Nous montrons que cet effet varie significativement selon que les grèves s'accompagnent ou non d'un degré élevé d'expression individuelle de conflit de la part des salariés. Nous mettons en évidence que les grèves n'ont d'effet positif sur la productivité 
du travail seulement lorsqu'elles ne s'accompagnent pas d'un problème d'absentéisme dans l'entreprise. Cet effet s'avère être croissant jusqu'à 5 grèves sur la période 2002-2004, puis décroissant au-delà de ce seuil. Lorsqu'elles s'accompagnent d'un tel problème d'absentéisme, les grèves ne présentent aucun effet sur la productivité du travail. Ces derniers résultats conduisent à amender l'analyse faite des grèves comme formes d'expression collective (e.g. Godard 1992 ; Knight 1989), bénéfiques pour la productivité du travail. Nous montrons, en effet, que l'effet 'thérapeutique' de la grève (Drinkwater et Ingram 2005) n'opère pleinement que si les salariés décident de traduire leur insatisfaction dans des revendications et d'utiliser la grève pour exprimer leurs revendications. Si, à l'inverse, les salariés ont recours l'absentéisme pour signifier leur insatisfaction, il est très vraisemblable que la productivité du travail soit déjà à un niveau faible. Dans ce cadre, le recours à la grève n'aurait pas de conséquence positive sur la productivité.

\section{Conclusion}

Nous estimons, dans cet article, l'effet de la fréquence des grèves sur la productivité du travail dans le cadre des entreprises françaises, à partir des données appariées de l'enquête REPONSE 2004-2005 et des Enquêtes Annuelles d'Entreprises (EAE). L'effet de l'activité de grève sur la productivité des entreprises a principalement été étudié par le passé dans le cadre des pays anglo-saxons. Aucune étude, à ce jour, n'a été réalisée dans le contexte français, malgré le développement de données microéconomiques détaillées dans ce domaine. La littérature existante suppose l'existence d'effets indirects des grèves sur la productivité du travail, relatifs à l'expression individuelle de conflit des salariés sur le lieu de travail. Ces effets indirects ont été peu évalués empiriquement. Dans cet article, nous proposons d'évaluer dans quelle mesure l'effet de la fréquence des grèves sur la productivité du travail varie en fonction de l'absentéisme des salariés dans l'entreprise.

Les résultats présentés dans cet article révèlent un effet non-linéaire de la fréquence des grèves sur la productivité du travail dans les entreprises françaises, conformément à des résultats présentés pour l'industrie manufacturière britannique (Knight 1989). En contrôlant les biais statistiques liés à l'hétérogénéité inobservée, grâce à une approche par fonction de contrôle, nous obtenons que les grèves ont un effet positif et croissant sur la productivité du travail jusqu'à cinq grèves au cours de la période 2002-2004, puis neutre au-delà. Cet effet positif d'une fréquence modérée des grèves sur la productivité du travail peut s'interpréter à travers leur rôle d'expression collective - ou 'collective voice' (e.g. Drinkwater et Ingram 2005 ; Godard 1992; Knight 1989) -, tel un moyen efficace de communication dans l'entreprise, améliorant en outre le 'moral' et la coopération des salariés.

Nous mettons, ensuite, en évidence que l'effet des grèves sur la productivité du travail varie significativement selon qu'il existe ou non un problème d'absentéisme dans l'entreprise, en contrôlant les biais d'endogénéité et de sélection endogène. La productivité du travail s'avère être positivement influencée par la fréquence des grèves uniquement lorsque l'absentéisme n'est pas un problème dans l'entreprise. Sinon, les grèves ne présentent pas d'effet significatif sur la productivité du travail. Nous soulignons ainsi que le rôle positif du mécanisme d'expression collective fourni par la grève ne joue pleinement que lorsque les salariés la privilégient pour exprimer leur insatisfaction. Lorsque les salariés tendent à emprunter, parallèlement à la 
grève, des formes individuelles de conflit, tel l'absentéisme, ce rôle thérapeutique des grèves n’apparaît plus être à l'oeuvre.

L'analyse empirique, conduite dans cet article, considère essentiellement une relation statique entre la fréquence des grèves et la productivité du travail. Cette analyse mériterait d'être approfondie en tenant compte des relations dynamiques entre les grèves et la productivité du travail. Les effets 'potentiellement' indirects de la grève, présentés dans cet article, nécessiteraient ainsi d'être examinés dans le temps, à partir de données longitudinales sur ces formes de conflit.

\section{Références}

Amiti, M. et Wei, S.-J. (2009). Service Offshoring and Productivity : Evidence from the US. World Economy, 32(2) :203-220.

Amossé, T. (2006). Le dialogue social en entreprise : une intensification de l'activité institutionnelle, des salariés faiblement engagés. Premières Synthèses, $N^{\circ} 39.1$ (Dares).

Amossé, T. et JACOD, O. (2008). Salariés, représentants du personnel et directions : quelles interactions entre les acteurs des relations sociales en entreprise? In Amossé, T., BLOCHLondon, C. et WolfF, L., éditeurs : Les relations sociales en entreprise. Un portrait à partir des enquêtes "Relations professionnelles et négociations d'entreprise", pages 165-191, Paris. La Découverte "Recherches".

Ananian, S. et Aubert, P. (2006). Travailleurs âgés, nouvelles technologies et changements organisationnels : un réexamen à partir de l'enquête REPONSE. Economie et Statistique, $397: 21-49$.

Bemmels, B. (1987). How Unions Affect Productivity in Manufacturing Plants. Industrial and Labor Relations Review, 40(2) :241-253.

Béroud, S., Denis, J.-M., Desage, G., Giraud, B. et Pélisse, J. (2008). Entre grèves et conflits : les luttes quotidiennes au travail. Rapport de recherche $\mathrm{N}^{\circ} 49$, Centre d'Etudes de l'Emploi (CEE), Noisy-le-Grand.

Besancenot, D. et Vranceanu, R. (1999). A Trade Union Model with Endogenous Militancy : Interpreting the French Case. Labour Economics, 6(3) :355-373.

Black, S. E. et Lynch, L. M. (2001). How to Compete : The Impact of Workplace Practices and Information Technology on Productivity. The Review of Economics and Statistics, 83(3) :434-445.

Breda, T. (2008). Union Wage Differentials : What About France? In European Association of Labour Economics Conference Paper, Amsterdam.

Brochard, D. (2003). Évaluation des statistiques administratives sur les conflits du travail. Document d'études $\mathrm{N}^{\circ} 79$, Dares.

Buffeteau, S. et Essafi, C. A. (2006). L'activité féminine en france : quelles évolutions récentes, quelles tendances pour l'avenir ? Economie et statistique, 398(1) :85-97. 
Carlier, A. (2008). Mesurer les grèves dans les entreprises : des données administratives aux données d'enquêtes. Document d'études N 139, Dares.

Carlier, A. et Tenret, E. (2007). Des conflits du travail plus nombreux et plus diversifiés. Premières Synthèses, $\mathrm{N}^{\circ}$ 08.1(Dares).

Coutrot, T. (1996). Relations sociales et performance économique, une première analyse empirique du cas français. Travail et Emploi, 66 :39-58.

DeVaro, J. (2008). The Effects of Self-Managed and Closely Managed Teams on Labor Productivity and Product Quality : An Empirical Analysis of a Cross-Section of Establishments. Industrial Relations : A Journal of Economy and Society, 47(4) :659-697.

Drinkwater, S. et Ingram, P. (2005). Have Industrial Relations in the UK Really Improved? Labour, 19(2) :373-398.

FAIRris, D. (1998). Social Stratification, Endogenous Contradictions, and Institutional Change. Eastern Economic Journal, 24(3) :311-324.

Farrell, D. et Rusbult, C. E. (1992). Exploring the Exit, Voice, Loyalty, and Neglect Typology : The Influence of Job Satisfaction, Quality of Alternatives, and Investment Size. Employee Responsibilities and Rights Journal, 5(3) :201-218.

Flaherty, S. (1987). Strike Activity, Worker Militancy, and Productivity Change in Manufacturing, 1961-1981. Industrial and Labor Relations Review, 40(4) :585-600.

Freeman, R. B. et Medoff, J. L. (1979). The Two Faces of Unionism. Public Interest, 57 :69-93.

Freeman, R. B. et Medoff, J. L. (1984). What Do Unions Do? Basic Books, New York.

Ghinetti, P. (2004). Does Education Matter? Schooling Endogeneity and the Public Wage Premium in Italy. Discussion Paper 39, DISCE - Quaderni dell'Istituto di Economia dell'Impresa e del Lavoro, Università Cattolica, Milan.

GodArd, J. (1992). Strikes as Collective Voice : A Behavioral Analysis of Strike Activity. Industrial and Labor Relations Review, 46(1) :161-175.

Gramm, C. L. (1986). The Determinants of Strike Incidence and Severity : A Micro-Level Study. Industrial and Labor Relations Review, 39(3):361-376.

Green, F. (2004). Why Has Work Effort Become More Intense? Industrial Relations : A Journal of Economy and Society, 43(4) :709-741.

Groux, G. et Pernot, J.-M. (2008). La grève. Presses de Sciences Po, Paris.

Haltiwanger, J. C., Lane, J. I. et Spletzer, J. R. (1999). Productivity Differences across Employers : The Roles of Employer Size, Age, and Human Capital. The American Economic Review, 89(2) :94-98.

Hebdon, R. P. et Stern, R. N. (1998). Tradeoffs among Expressions of Industrial Conflict : Public Sector Strike Bans and Grievance Arbitrations. Industrial and Labor Relations Review, $51(2): 204-221$. 
Heckman, J. J. et RobB, R. (1985). Alternative Methods for Evaluating the Impact of Interventions : An Overview. Journal of Econometrics, 30(1-2) :239-267.

Hyman, R. (1972). Strikes. Fontana/Collins, London.

IChniowski, C. (1986). The Effects of Grievance Activity on Productivity. Industrial and Labor Relations Review, 40(1) :75-89.

Jensen, J. B., McGuckin, R. H. et Stiroh, K. J. (2001). The Impact of Vintage and Survival on Productivity : Evidence from Cohorts of U.S. Manufacturing Plants. The Review of Economics and Statistics, 83(2) :323-332.

Katz, H. C., Kochan, T. A. et Gobeille, K. R. (1983). Industrial Relations Performance, Economic Performance and QWL Programs : An Interplant Analysis. Industrial and Labor Relations Review, $37(1): 3-17$.

Kleiner, M. M., Leonard, J. S. et Pilarski, A. M. (2002). How Industrial Relations Affects Plant Performance : The Case of Commercial Aircraft Manufacturing. Industrial and Labor Relations Review, 55(2) :195-218.

Kleiner, M. M., Nickelsburg, G. et Pilarski, A. (1995). Monitoring, Grievances, and Plant Performance. Industrial Relations : A Journal of Economy and Society, 34(2) :169-189.

Knight, K. G. (1989). Labour Productivity and Strike Activity in British Manufacturing Industries : Some Quantitative Evidence. British Journal of Industrial Relations, 27(3) :365-374.

Krueger, A. B. et Mas, A. (2004). Strikes, Scabs, and Tread Separations : Labor Strife and the Production of Defective Bridgestone/Firestone Tires. Journal of Political Economy, 112(2) :253289.

Mas, A. (2008). Labour Unrest and the Quality of Production : Evidence from the Construction Equipment Resale Market. Review of Economic Studies, 75(1) :229-258.

Matteucci, N., O’Mahony, M., Robinson, C. et Zwick, T. (2005). Productivity, Workplace Performance and ICT : Industry and Firm-Level Evidence for Europe and the US. Scottish Journal of Political Economy, 52(3):359-386.

McHugh, R. (1991). Productivity Effects of Strikes in Struck and Nonstruck Industries. Industrial and Labor Relations Review, 44(4) :722-732.

Mefford, R. N. (1986). The Effect of Unions on Productivity in a Multinational Manufacturing Firm. Industrial and Labor Relations Review, 40(1) :105-114.

Naples, M. I. (1988). Industrial Conflict, the Quality of Worklife, and the Productivity Slowdown in U.S. Manufacturing. Eastern Economic Journal, 14(2) :157-166.

Nicolitsas, D. (2000). Accounting for Strikes : Evidence from UK Manufacturing in the 1980s. Labour, 14(3) :417-440.

Norsworthy, J. R. et Zabala, C. A. (1985). Worker Attitudes, Worker Behavior, and Productivity in the U.S. Automobile Industry, 1959-1976. Industrial and Labor Relations Review, 38(4):544-557. 
Pernot, J.-M. (2005). Conflits du public, conflits du privé. In Denis, J.-M., éditeur : Le Conflit en grève ? Tendances et perspectives de la conflictualité contemporaine, pages 147-169, Paris. La Dispute.

Roodman, D. M. (2011). CMP : Stata module to implement conditional (recursive) mixed process estimator. Statistical Software Components, Boston College Department of Economics.

Sapsford, D. et Turnbull, P. (1994). Strikes and Industrial Conflict in Britain's Docks : Balloons or Icebergs? Oxford Bulletin of Economics and Statistics, 56(3) :249-265.

Scheuer, S. (2006). A Novel Calculus? Institutional Change, Globalization and Industrial Conflict in Europe. European Journal of Industrial Relations, 12(2) :143-164.

Sirot, S. (2002). La grève en France : une histoire sociale (XIX $X^{e}-X X^{e}$ siècle). Collection "Histoire". Odile Jacob, Paris.

Syverson, C. (2004). Product Substitutability and Productivity Dispersion. The Review of Economics and Statistics, 86(2):534-550.

Tanguy, J. (2013). Collective and Individual Conflicts in the Workplace : Evidence from France. Industrial Relations : A Journal of Economy and Society, 52(1) :102-133.

Tracy, J. S. (1986). An Investigation into the Determinants of U.S. Strike Activity. The American Economic Review, 76(3) :423-436.

Vella, F. et Verbeek, M. (1999). Estimating and Interpreting Models with Endogenous Treatment Effects. Journal of Business \& Economic Statistics, 17(4) :473-478.

Zabel, J. E. (1998). An Analysis of Attrition in the Panel Study of Income Dynamics and the Survey of Income and Program Participation with an Application to a Model of Labor Market Behavior. The Journal of Human Resources, 33(2) :479-506.

Zwick, T. (2004). Employee Participation and Productivity. Labour Economics, 11(6) :715-740. 
TABLE 7 - Définition des variables

\begin{tabular}{|c|c|c|c|}
\hline Variables & Définition & Moy. & E.-type \\
\hline Valeur ajoutée $(Y)$ & $\begin{array}{l}\text { Valeur ajoutée déflatée par les indices de prix sectoriels publiés par l'IN- } \\
\text { SEE }\end{array}$ & 438668 & 2016857 \\
\hline Nombre total de salariés $(L)$ & Nombre total de salariés dans l'entreprise en 2004 & 4926.64 & 16469.96 \\
\hline Productivité du travail $(\ln y)$ & Logarithme du ratio valeur ajoutée sur nombre total de salariés & 3.925 & 0.635 \\
\hline Stock de capital $(K)$ & $\begin{array}{l}\text { Le stock de capital est déterminé à partir de la valeur comptable des } \\
\text { immobilisations corporelles en } 2004\end{array}$ & 2434799 & $1.74 \times 10^{7}$ \\
\hline $\begin{array}{l}\text { Intensité } \quad \text { capitalistique } \\
(\ln K / L)\end{array}$ & $\begin{array}{l}\text { Logarithme du ratio entre le stock de capital et le nombre total de salariés } \\
\text { dans l'entreprise, en } 2004\end{array}$ & 3.756 & 1.754 \\
\hline Taille d'entreprise $(\ln L)$ & Logarithme du nombre total de salariés dans l'entreprise en 2004 & 6.273 & 2.030 \\
\hline Secteur & $\begin{array}{l}\text { Variables binaires égales à } 1 \text { si l'entreprise appartient à l'un des } 16 \text { postes } \\
\text { de la Nomenclature d'Activités Française (NAF), égales à } 0 \text { sinon }\end{array}$ & - & - \\
\hline Age de l'entreprise & $\begin{array}{l}\text { Variables binaires égales à } 1 \text { si l'entreprise appartient à une des six classes } \\
\text { suivantes : moins de } 10 \text { ans, } 10-14 \text { ans, } 15-19 \text { ans, } 20-29 \text { ans, 30-44 ans, } \\
45 \text { ans et plus, égales à } 0 \text { sinon }\end{array}$ & - & - \\
\hline Multi-établissements & $\begin{array}{l}\text { Variable binaire égale à } 1 \text { si l'entreprise est composée d'au moins deux } \\
\text { établissements, égale à } 0 \text { sinon }\end{array}$ & \multicolumn{2}{|c|}{0.626} \\
\hline Bourse & $\begin{array}{l}\text { Variable binaire égale à } 1 \text { si l'entreprise est cotée en Bourse, égale à } 0 \\
\text { sinon }\end{array}$ & \multicolumn{2}{|c|}{0.420} \\
\hline$\%$ cadres $^{\mathrm{a}}$ & & 0.159 & 0.202 \\
\hline$\% \mathrm{TAM}^{\mathrm{b}}$ & Variables continues indiquant le pourcentage de la main d'oeuvre & 0.208 & 0.164 \\
\hline \% employés & appartenant à chacune des catégories socio-professionnelles, en 2004 & 0.202 & 0.271 \\
\hline$\%$ ouvriers & & 0.431 & 0.324 \\
\hline$\%$ femmes & $\begin{array}{l}\text { Variable continue indiquant le pourcentage de salariées au sein de la main } \\
\text { d'oeuvre }\end{array}$ & 0.327 & 0.229 \\
\hline$\% \mathrm{CDD}$ & $\begin{array}{l}\text { Variable continue indiquant le pourcentage de salariés avec un contrat à } \\
\text { durée déterminée au sein de la main d'oeuvre }\end{array}$ & 0.048 & 0.111 \\
\hline Fréquence des grèves & Nombre de grèves déclarées sur la période 2002-2004:0, 1-2, 3-5, > 5 & - & - \\
\hline Présence syndicale & $\begin{array}{l}\text { Variable binaire égale à } 1 \text { si au moins un délégué syndical est présent dans } \\
\text { l'établissement, égale à } 0 \text { sinon }\end{array}$ & \multicolumn{2}{|c|}{0.680} \\
\hline Absentéisme & $\begin{array}{l}\text { Variable binaire égale à } 1 \text { si l'employeur considère que l'absentéisme re- } \\
\text { présente un problème pour au moins une catégorie socio-professionnelle } \\
\text { (cadres, techniciens et agents de maîtrise, employés, ouvriers) dans l'éta- } \\
\text { blissement, égale à } 0 \text { sinon }\end{array}$ & \multicolumn{2}{|c|}{0.531} \\
\hline Taux de syndicalisation $>5 \%$ & $\begin{array}{l}\text { Variable binaire égale à } 1 \text { si le taux de syndicalisation des salariés de } \\
\text { l'établissement, égale à } 0 \text { sinon }\end{array}$ & \multicolumn{2}{|c|}{0.460} \\
\hline $\begin{array}{l}\text { Faible activité syndicale (bas- } \\
\text { sin d'emploi) }\end{array}$ & $\begin{array}{l}\text { Variable binaire égale à } 1 \text { si l'activité syndicale dans le bassin d'emploi } \\
\text { est jugée comme 'peu intense' ou 'très peu intense' par l'employeur, égale } \\
\text { à } 0 \text { sinon }\end{array}$ & \multicolumn{2}{|c|}{0.497} \\
\hline CFDT & $\begin{array}{l}\text { Variable binaire égale à } 1 \text { si la CFDT est représentée par un ou plusieurs } \\
\text { délégués syndicaux dans l'entreprise, égale à } 0 \text { sinon }\end{array}$ & \multicolumn{2}{|c|}{0.577} \\
\hline CGT & $\begin{array}{l}\text { Variable binaire égale à } 1 \text { si la CGT est représentée par un ou plusieurs } \\
\text { délégués syndicaux dans l'entreprise, égale à } 0 \text { sinon }\end{array}$ & \multicolumn{2}{|c|}{0.585} \\
\hline
\end{tabular}

Notes : Echantillon de 2149 observations. ${ }^{\text {a }}$ Les cadres intègrent également les dirigeants de l'entreprise. ${ }^{\text {b }}$ TAM : Techniciens et Agents de Maîtrise. 


\section{Annexe}

\section{Calcul des termes de correction dans le modèle en deux étapes : contrôle de l'auto-sélection de l'absentéisme et de l'endogénéité des fréquences de grèves (Section 5)}

Nous détaillons, dans ce document annexe, la procédure permettant d'obtenir les termes de correction $\lambda_{1}$ et $\lambda_{2}$ introduits dans l'équation (13) afin de contrôler simultanément l'autosélection liée à l'absentéisme $k=0,1$ et l'endogénéité des fréquences de grèves dans l'équation de productivité du travail, en tenant compte de la corrélation non-nulle entre l'absentéisme et la fréquence des grèves. La méthode d'estimation proposée est une méthode en deux étapes : en première étape, les équations d'absentéisme $\left(A^{*}\right)$ et de fréquence des grèves $\left(S^{*}\right)$ sont estimées simultanément dans un modèle de type bivarié :

$$
\begin{aligned}
& S_{i}^{*}=\mathbf{W}_{i}^{\prime} \theta+\nu_{i}, \quad S_{i}=j \text { si } \mu_{j-1}<S_{i}^{*} \leqslant \mu_{j}, \quad j \in\{0,3\} \\
& A^{*}=\sum_{j=1}^{3} \zeta_{j} S_{i j}+\mathbf{V}_{i}^{\prime} \gamma+\eta_{i}, \quad A=1\left(A^{*}>0\right)
\end{aligned}
$$

où $\rho=\operatorname{corr}(\nu, \eta)$ est supposé non-nul. Les paramètres estimés lors de cette première étape $\{\hat{\theta}, \hat{\gamma}, \hat{\rho}\}$ sont utilisés pour calculer les termes de correction $\lambda_{1}$ et $\lambda_{2}$, introduits dans l'équation structurelle de productivité du travail $(\ln y)$, en deuxième étape :

$$
\ln y_{k i}=\mathbf{X}_{i}^{\prime} \beta_{k}+\sum_{j=1}^{3} \delta_{k j} S_{i j}+\sigma_{k \nu} \lambda_{1 k i}+\sigma_{k \eta} \lambda_{2 k i}+u_{k i} ; \quad k=0,1
$$

Nous détaillons les calculs permettant d'obtenir les termes de correction $\lambda_{1}$ et $\lambda_{2}$ dans le cas où $k=1$, i.e. l'effet de la fréquence des grèves sur la productivité du travail est estimé pour les seuls établissements déclarant un problème d'absentéisme de leurs salariés. Cette procédure est également détaillée dans les travaux de Zabel (1998) et Ghinetti (2004) .

En considérant l'équation de productivité du travail pour les entreprises concernées par un problème d'absentéisme $(A=1)$, l'espérance conditionnelle du terme d'erreur $u_{1}$ peut s'écrire et se décomposer ainsi :

$$
\begin{aligned}
E\left(u_{1} \mid S, A=1\right) & =\sum_{j=0}^{3} E\left(u_{1} \mid S=j, A=1\right) \times(S=j) \\
& =E\left(u_{1} \mid \nu<\mu_{1}-\mathbf{W}^{\prime} \theta ; \eta>-\mathbf{V}^{\prime} \gamma\right) \times(S=0) \\
& +\sum_{j=1}^{2} E\left(u_{1} \mid \mu_{j}-\mathbf{W}^{\prime} \theta<\nu<\mu_{j+1}-\mathbf{W}^{\prime} \theta ; \eta>-\mathbf{V}^{\prime} \gamma\right) \times(S=j) \\
& +E\left(u_{1} \mid \nu>\mu_{3}-\mathbf{W}^{\prime} \theta ; \eta>-\mathbf{V}^{\prime} \gamma\right) \times(S=3)
\end{aligned}
$$

Compte tenu des hypothèses de distribution retenues : 


$$
\begin{aligned}
& E\left(u_{1} \mid \nu, \eta\right)=c_{1} \nu+c_{2} \eta \\
& c_{1}=\frac{\sigma_{1 \nu}-\sigma_{1 \eta}}{1-\rho} ; \quad c_{2}=\frac{\sigma_{1 \eta}-\sigma_{1 \nu}}{1-\rho}
\end{aligned}
$$

nous pouvons ensuite dériver la forme fonctionnelle de ces espérances conditionnelles.

Lorsque $\mathbf{S}=\mathbf{0}$, la forme fonctionnelle de l'espérance conditionnelle de $u_{1}$ peut s'écrire ainsi :

$$
\begin{aligned}
E\left(u_{1} \mid \nu<\mu_{1}-\mathbf{W}^{\prime} \theta, \eta>-\mathbf{V}^{\prime} \gamma\right) & =c_{1} E\left(\nu \mid \nu<\mu_{1}-\mathbf{W}^{\prime} \theta ; \eta>-\mathbf{V}^{\prime} \gamma\right) \\
& +c_{2} E\left(\eta \mid \nu<\mu_{1}-\mathbf{W}^{\prime} \theta ; \eta>-\mathbf{V}^{\prime} \gamma\right)
\end{aligned}
$$

En utilisant les résultats des distributions normales bivariées tronquées, nous obtenons :

$$
\begin{aligned}
& c_{1} E\left(\nu \mid \nu<\mu_{1}-\mathbf{W}^{\prime} \theta ; \eta>-\mathbf{V}^{\prime} \gamma\right)=-c_{1} \lambda_{01}^{1}+\rho c_{1} \lambda_{01}^{2} \\
& \text { et } \\
& c_{2} E\left(\eta \mid \nu<\mu_{1}-\mathbf{W}^{\prime} \theta ; \eta>-\mathbf{V}^{\prime} \gamma\right)=c_{2} \lambda_{01}^{2}-\rho c_{2} \lambda_{01}^{1}
\end{aligned}
$$

avec :

$$
\begin{aligned}
& \lambda_{01}^{1}=\frac{\phi\left(\mu_{1}-\mathbf{W}^{\prime} \theta\right) \cdot\left[1-\Phi\left(\frac{-\mathbf{V}^{\prime} \gamma-\rho\left(\mu_{1}-\mathbf{W}^{\prime} \theta\right)}{\sqrt{1-\rho^{2}}}\right)\right]}{\operatorname{Pr}\left(\nu<\mu_{1}-\mathbf{W}^{\prime} \theta ; \eta>-\mathbf{V}^{\prime} \gamma\right)} \\
& \lambda_{01}^{2}=\frac{\phi\left(-\mathbf{V}^{\prime} \gamma\right) \cdot\left[1-\Phi\left(\frac{\mu_{1}-\mathbf{W}^{\prime} \theta+\rho \mathbf{V}^{\prime} \gamma}{\sqrt{1-\rho^{2}}}\right)\right]}{\operatorname{Pr}\left(\nu<\mu_{1}-\mathbf{W}^{\prime} \theta ; \eta>-\mathbf{V}^{\prime} \gamma\right)}
\end{aligned}
$$

où :

$$
\operatorname{Pr}(S=0, A=1)=\operatorname{Pr}\left(\nu<\mu_{1}-\mathbf{W}^{\prime} \theta ; \eta>-\mathbf{V}^{\prime} \gamma\right)=\Phi_{2}\left(\mathbf{V}^{\prime} \gamma, \mu_{1}-\mathbf{W}^{\prime} \theta,-\rho\right)
$$

$\Phi_{2}$ correspond à la fonction de distribution cumulative de la loi normale bivariée. Ainsi, l'espérance conditionnelle du terme d'erreur $u_{1}$ lorsque $A=1$ et $S=0$ s'obtient en ajoutant les termes :

$$
E\left(u_{1} \mid \nu<\mu_{1}-\mathbf{W}^{\prime} \theta, \eta>-\mathbf{V}^{\prime} \gamma\right)=-\sigma_{a_{1} \nu} \lambda_{01}^{1}+\sigma_{1 \eta} \lambda_{01}^{2}
$$

Lorsque $\mathbf{S}=\mathbf{j}, \forall j \in\{1,2\}$, la forme fonctionnelle des espérances conditionnelles peut s'écrire ainsi : 


$$
\begin{aligned}
& E\left(u_{1} \mid \mu_{j}-\mathbf{W}^{\prime} \theta<\nu<\mu_{j+1}-\mathbf{W}^{\prime} \theta ; \eta>-\mathbf{V}^{\prime} \gamma\right) \\
= & c_{1} E\left(\nu \mid \mu_{j}-\mathbf{W}^{\prime} \theta<\nu<\mu_{j+1}-\mathbf{W}^{\prime} \theta ; \eta>-\mathbf{V}^{\prime} \gamma\right) \\
+ & c_{2} E\left(\eta \mid \mu_{j}-\mathbf{W}^{\prime} \theta<\nu<\mu_{j+1}-\mathbf{W}^{\prime} \theta ; \eta>-\mathbf{V}^{\prime} \gamma\right)
\end{aligned}
$$

À partir des résultats des distributions normales bivariées tronquées, nous obtenons ainsi :

$$
\begin{aligned}
& c_{1} E\left(\nu \mid \mu_{j}-\mathbf{W}^{\prime} \theta<\nu<\mu_{j+1}-\mathbf{W}^{\prime} \theta ; \eta>-\mathbf{V}^{\prime} \gamma\right)=c_{1}\left(\lambda_{j 1}^{1}-\lambda_{j 1}^{2}\right)+\rho c_{1} \lambda_{j 1}^{3} \\
& \text { et } \\
& c_{2} E\left(\eta \mid \mu_{j}-\mathbf{W}^{\prime} \theta<\nu<\mu_{j+1}-\mathbf{W}^{\prime} \theta ; \eta>-\mathbf{V}^{\prime} \gamma\right)=c_{2} \rho\left(\lambda_{j 1}^{1}-\lambda_{j 1}^{2}\right)+c_{2} \lambda_{j 1}^{3}
\end{aligned}
$$

avec :

$$
\begin{aligned}
\lambda_{j 1}^{1}= & \frac{\phi\left(\mu_{j}-\mathbf{W}^{\prime} \theta\right) \cdot\left[1-\Phi\left(\frac{-\mathbf{V}^{\prime} \gamma-\rho\left(\mu_{j}-\mathbf{W}^{\prime} \theta\right)}{\sqrt{1-\rho^{2}}}\right)\right]}{\operatorname{Pr}\left(\mu_{j}-\mathbf{W}^{\prime} \theta<\nu<\mu_{j+1}-\mathbf{W}^{\prime} \theta ; \eta>-\mathbf{V}^{\prime} \gamma\right)} \\
\lambda_{j 1}^{2}= & \frac{\phi\left(\mu_{j+1}-\mathbf{W}^{\prime} \theta\right) \cdot\left[1-\Phi\left(\frac{-\mathbf{V}^{\prime} \gamma-\rho\left(\mu_{j+1}-\mathbf{W}^{\prime} \theta\right)}{\sqrt{1-\rho^{2}}}\right)\right]}{\operatorname{Pr}\left(\mu_{j}-\mathbf{W}^{\prime} \theta<\nu<\mu_{j+1}-\mathbf{W}^{\prime} \theta ; \eta>-\mathbf{V}^{\prime} \gamma\right)} \\
\lambda_{j 1}^{3}= & \frac{\phi\left(-\mathbf{V}^{\prime} \gamma\right) \cdot\left[\Phi\left(\frac{\mu_{j+1}-\mathbf{W}^{\prime} \theta+\rho \mathbf{V}^{\prime} \gamma}{\sqrt{1-\rho^{2}}}\right)-\Phi\left(\frac{\mu_{j}-\mathbf{W}^{\prime} \theta-\rho \mathbf{V}^{\prime} \gamma}{\sqrt{1-\rho^{2}}}\right)\right]}{\operatorname{Pr}\left(\mu_{j}-\mathbf{W}^{\prime} \theta<\nu<\mu_{j+1}-\mathbf{W}^{\prime} \theta ; \eta>-\mathbf{V}^{\prime} \gamma\right)}
\end{aligned}
$$

où :

$$
\begin{aligned}
\operatorname{Pr}(S=j, A=1) & =\operatorname{Pr}\left(\mu_{j}-\mathbf{W}^{\prime} \theta<\nu<\mu_{j+1}-\mathbf{W}^{\prime} \theta ; \eta>-\mathbf{V}^{\prime} \gamma\right) \\
& =\Phi_{2}\left(\mathbf{V}^{\prime} \gamma, \mu_{j+1}-\mathbf{W}^{\prime} \theta,-\rho\right)-\Phi_{2}\left(\mathbf{V}^{\prime} \gamma, \mu_{j}-\mathbf{W}^{\prime} \theta,-\rho\right)
\end{aligned}
$$

L'espérance conditionnelle du terme d'erreur $u_{1}$, lorsque $A=1$ et $S=j, \forall j \in\{1,2\}$, s'obtient en combinant les termes précédents :

$$
E\left(u_{1} \mid \mu_{j}-\mathbf{W}^{\prime} \theta<\nu<\mu_{j+1}-\mathbf{W}^{\prime} \theta ; \eta>-\mathbf{V}^{\prime} \gamma\right)=\sigma_{1 \nu}\left(\lambda_{j 1}^{1}-\lambda_{j 1}^{2}\right)+\sigma_{1 \eta} \lambda_{j 1}^{3}
$$

Enfin, lorsque $\mathbf{S}=\mathbf{3}$, la forme fonctionnelle de l'espérance conditionnelle s'écrit :

$$
\begin{aligned}
E\left(u_{1} \mid \nu>\mu_{3}-\mathbf{W}^{\prime} \theta ; \eta>-\mathbf{V}^{\prime} \gamma\right) & =c_{1} E\left(\nu \mid \nu>\mu_{3}-\mathbf{W}^{\prime} \theta ; \eta>-\mathbf{V}^{\prime} \gamma\right) \\
& +c_{2} E\left(\eta \mid \nu>\mu_{3}-\mathbf{W}^{\prime} \theta ; \eta>-\mathbf{V}^{\prime} \gamma\right)
\end{aligned}
$$

À partir des résultats des distributions normales bivariées tronquées, nous obtenons ainsi : 


$$
\begin{aligned}
& \quad c_{1} E\left(\nu \mid \nu>\mu_{3}-\mathbf{W}^{\prime} \theta ; \eta>-\mathbf{V}^{\prime} \gamma\right)=c_{1} \lambda_{31}^{1}+\rho c_{1} \lambda_{31}^{2} \\
& \text { et } \\
& \qquad c_{2} E\left(\eta \mid \nu>\mu_{3}-\mathbf{W}^{\prime} \theta ; \eta>-\mathbf{V}^{\prime} \gamma\right)=c_{2} \rho \lambda_{31}^{2}+c_{2} \lambda_{31}^{1}
\end{aligned}
$$

avec :

$$
\begin{aligned}
\lambda_{31}^{1}= & \frac{\phi\left(\mu_{3}-\mathbf{W}^{\prime} \theta\right) \cdot\left[1-\Phi\left(\frac{-\mathbf{V}^{\prime} \gamma-\rho\left(\mu_{3}-\mathbf{W}^{\prime} \theta\right)}{\sqrt{1-\rho^{2}}}\right)\right]}{\operatorname{Pr}\left(\nu>\mu_{3}-\mathbf{W}^{\prime} \theta ; \eta>-\mathbf{V}^{\prime} \gamma\right)} \\
\lambda_{31}^{2}= & \frac{\phi\left(-\mathbf{V}^{\prime} \gamma\right) \cdot\left[1-\Phi\left(\frac{\mu_{3}-\mathbf{W}^{\prime} \theta+\rho \mathbf{V}^{\prime} \gamma}{\sqrt{1-\rho^{2}}}\right)\right]}{\operatorname{Pr}\left(\nu>\mu_{3}-\mathbf{W}^{\prime} \theta ; \eta>-\mathbf{V}^{\prime} \gamma\right)}
\end{aligned}
$$

où :

$$
\operatorname{Pr}\left(\nu>\mu_{3}-\mathbf{W}^{\prime} \theta ; \eta>-\mathbf{V}^{\prime} \gamma\right)=\operatorname{Pr}(S=3, A=1)=\Phi_{2}\left(\mathbf{V}^{\prime} \gamma, \mathbf{W}^{\prime} \theta-\mu_{3}, \rho\right)
$$

L'espérance conditionnelle de $u_{1}$ lorsque $A=1$ et $S=3$, s'obtient en combinant les termes précédents :

$$
E\left(u_{1} \mid \nu>\mu_{3}-\mathbf{W}^{\prime} \theta, \eta>-\mathbf{V}^{\prime} \gamma\right)=\sigma_{1 \nu} \lambda_{31}^{1}+\sigma_{1 \eta} \lambda_{31}^{2}
$$

Les deux termes de correction $\lambda_{11}$ et $\lambda_{21}$ introduits dans l'équation structurelle de productivité du travail (13), pour $k=1$, sont finalement déterminés à partir des résultats précédents :

$$
\begin{aligned}
& \lambda_{11}=-\lambda_{01}^{1} \times(S=0)+\left(\lambda_{11}^{1}-\lambda_{11}^{2}\right) \times(S=1)+\left(\lambda_{21}^{1}-\lambda_{21}^{2}\right) \times(S=2)+\lambda_{31}^{1} \times(S=3) \\
& \lambda_{21}=\lambda_{01}^{2} \times(S=0)+\lambda_{11}^{3} \times(S=1)+\lambda_{21}^{3} \times(S=2)+\lambda_{31}^{2} \times(S=3)
\end{aligned}
$$

Cette procédure s'applique de façon similaire pour obtenir les termes de correction $\lambda_{10}$ et $\lambda_{20}$ dans le cas des entreprises ne déclarant pas de problème d'absentéisme $(A=0)$. 
14-3. Le non-recours au RSA "socle seul": L'hypothèse du patrimoine Sylvain Chareyron

14-2. Une évaluation de l'impact de l'aménagement des conditions de travail sur la reprise du travail après un cancer

Emmanuel Duguet, Christine Le Clainche

14-1. Renforcer la progressivité des prélèvements sociaux

Yannick L'Horty, Etienne Lehmann

\section{TEPP Rapports de Recherche 2013}

13-10. La discrimination à l'entrée des établissements scolaires privés : Les résultats d'une expérience contrôlée

Loïc du Parquet, Thomas Brodaty, Pascale Petit

13-9. Simuler les politiques locales favorisant l'accessibilité à l'emploi

Mathieu Bunel, Elisabeth Tovar

13-8. Le paradoxe des nouvelles politiques d'insertion

Jekaterina Dmitrijeva, Florent Fremigacci, Yannick L'Horty

13-7. L'emploi des seniors : un réexamen des écarts de taux d'emploi européens Laetitia Challe

13-6. Effets de quartier, effet de département : discrimination liée au lieu de résidence et accès à l'emploi

Pascale Petit, Mathieu Bunel, Emilia Ene Jones, Yannick L'Horty

13-5. Comment améliorer la qualité des emplois salariés exercés par les étudiants ? Les enseignements d'une expérience contrôlée

Jekaterina Dmitrijeva, Yannick L'Horty, Loïc Du Parquet, Pascale Petit

13-4. Evaluer l'efficacité d'une campagne de valorisation du bénévolat : Les enseignements de deux expériences contrôlées sur le marché du travail

Thomas Brodaty, Céline Emond, Yannick L’Horty, Loïc Du Parquet, Pascale Petit

13-3. Les différents parcours offerts par l'Education Nationale procurent-ils les mêmes chances d'accéder à l'emploi ?

Florent Fremigacci, Yannick L’Horty, Loïc Fu Parquet, Pascale Petit

13-2. Faut-il subventionner le permis de conduire des jeunes en difficulté d'insertion ?

Yannick L’Horty, Emmanuel Duguet, Pascale Petit, Bénédicte Rouland, Yiyi Tao

13-1. Anatomie d'une politique régionale de lutte contre les discriminations Yannick L'Horty 
La Fédération de recherche CNRS Travail, Emploi et Politiques Publiques (TEPP, FR $\mathbf{n}^{\circ} \mathbf{3 4 3 5}$ ) réunit des centres de recherche en économie et sociologie :

- Le Centre d'Etudes des Politiques Economiques de l'université d'Evry, EPEE, Université d'Evry Val d'Essonne

- Le Centre Pierre Naville, CPN, Université d'Evry Val d'Essonne

- Le Centre de Recherche en Economie et Management, CREM, Université de Caen Basse Normandie et Université de Rennes 1

- L'Equipe de Recherche sur les Marchés, l'Emploi et la Simulation, ERMES, Université deParis II Panthéon-Assas

- L'Equipe de Recherche sur l'Utilisation des Données Temporelles en Economie, ERUDITE, Université de Paris-Est Créteil et Université de Paris-Est Marne-la-Vallée

- Le Groupe d'Analyse des Itinéraires et des Niveaux Salariaux, GAINS, Université du Maine

La Fédération TEPP rassemble 150 chercheurs et enseignants-chercheurs, 140 doctorants et 40 chercheurs associés, qui étudient les mutations du travail et de l'emploi en relation avec les choix des entreprises et analysent les politiques publiques en mobilisant les nouvelles méthodes d'évaluation. 